\title{
EMPIRICAL ISOCHRONES FOR LOW MASS STARS IN NEARBY YOUNG ASSOCIATIONS
}

\author{
Gregory J. Herczeg (沈雷歌 $)^{1}$ AND Lynne A. HiLlennBRAND ${ }^{2}$ \\ ${ }^{1}$ Kavli Institute for Astronomy and Astrophysics, Peking University, Yi He Yuan Lu 5, Haidian Qu, 100871 Beijing, China \\ ${ }^{2}$ Caltech, MC105-24, 1200 E. California Blvd., Pasadena, CA 91125, USA \\ Received 2014 November 12; accepted 2015 May 21; published 2015 July 15
}

\begin{abstract}
Absolute ages of young stars are important for many issues in pre-main-sequence stellar and circumstellar evolution but long have been recognized as difficult to derive and calibrate. In this paper, we use literature spectral types and photometry to construct empirical isochrones in Hertzsprung-Russell diagrams for low mass stars and brown dwarfs in the $\eta \mathrm{Cha}, \epsilon$ Cha, and TW Hya Associations and the $\beta$ Pic and Tuc-Hor Moving Groups. A successful theory of premain-sequence evolution should match the shapes of the stellar loci for these groups of young stars. However, when comparing the combined empirical isochrones to isochrones predicted from evolutionary models, discrepancies lead to a spectral type (mass) dependence in stellar age estimates. Improved prescriptions for convection and boundary conditions in the latest models of pre-main-sequence evolution lead to a significantly improved correspondence between empirical and model isochrones, with small offsets at low temperatures that may be explained by observational uncertainties or by model limitations. Independent of model predictions, linear fits to combined stellar loci of these regions provide a simple empirical method to order clusters by luminosity with a reduced dependence on spectral type. Age estimates calculated from various sets of modern models that reproduce Li depletion boundary ages of the $\beta$ Pic Moving Group also imply a 4 Myr age for the low mass members of the Upper Sco OB Association, which is younger than the $11 \mathrm{Myr}$ age that has been recently estimated for intermediate and high mass members.
\end{abstract}

Key words: Hertzsprung-Russell and C-M diagrams - stars: evolution - stars: formation

\section{INTRODUCTION}

Age estimates for pre-main-sequence stars are critical in assessing the timescales for the formation of stellar systems and the evolution of molecular clouds. The fraction of embedded sources determines how long the dominant phase of stellar growth lasts and how quickly the envelope disperses. Similarly, when combined with disk fractions, age measurements place strict constraints on the time available for giant planets to form. Ages are also important in quantifying how the stellar contraction leads to rotational spin-up and changes in interior structure for solar and intermediate mass stars, factors that combine to determine the initial conditions for the evolution of magnetic activity for the duration of the stellar lifetime. Members of young stellar associations are usually dated by comparing their temperatures and luminosities to pre-main-sequence tracks. However, errors and biases in placing stars on HertzsprungRussell (HR) diagrams lead to uncertainty in ages of different clusters, while large luminosity spreads among members of individual clusters enhance uncertainty regarding age dating of any individual star (see the review by Soderblom et al. 2014).

Further uncertainties in pre-main-sequence age estimates from HR diagrams are traced directly to the varied stellar loci predicted from different stellar evolution models and to the lack of consistency between predicted and observed stellar loci. These differences have been noted between low and intermediate mass stars (e.g., Hillenbrand 1997; Hillenbrand et al. 2008; Naylor 2009) and among low mass stars of different masses (e.g., da Rio et al. 2010; Murphy et al. 2013; Alcala et al. 2014; Herczeg \& Hillenbrand 2014; Kraus et al. 2014; Malo et al. 2014). Components of young binary systems frequently have different estimates for age despite an expectation that they are co-eval (Hartigan et al. 1994; Hartigan \& Kenyon 2003; Kraus \& Hillenbrand 2009; Gennaro et al. 2012). As a direct consequence, age estimates depend on the considered range in spectral type or stellar mass.
Hillenbrand et al. (2008) found that in many regions G stars appear $\sim 2-5$ times older than $\mathrm{K}$ stars depending on which pre-main-sequence tracks were used. Pecaut et al. (2012) quantified these differences by measuring an age for the Upper Sco OB Association of $11 \pm 2 \mathrm{Myr}$, larger than the $5 \mathrm{Myr}$ age measured previously from pre-main-sequence low mass stars (Preibisch et al. 2002; Slesnick et al. 2006). Similarly, in both younger and older clusters, analyses of main sequence turnoff and intermediate mass stars in color-magnitude diagrams yield ages that are twice as large as those obtained from low mass pre-main-sequence stars (Mayne \& Naylor 2008; Naylor 2009; Bell et al. 2013).

Nearby stellar associations with ages of 5-40 Myr provide us with a laboratory in which to establish empirical isochrones that can be used as a model-independent clock and as a benchmark for testing pre-main-sequence models. Within a single association older than $10 \mathrm{Myr}$, the age spread expected from star formation processes is small compared to the absolute age. Member stars have traveled far from any remnant molecular material associated with their birth cloud and are unaffected by interstellar extinction. Most disks have already dispersed by these ages, so observational uncertainties related to accretion and circumstellar extinction are negligible for the vast majority of stars in this age range. In recent years, the growing number of proper motions and parallax distances have led to a substantial improvement in the accounting of membership within young associations (e.g., Malo et al. 2013; Kraus et al. 2014). The HR diagrams of these nearby moving groups should consequently have only a small age spread.

At younger ages, placing stars on HR diagrams is more problematic because accretion and extinction affect temperature and luminosity measurements and because the effect of any genuine age spread on luminosities decreases (in dex) with age. Nevertheless, wide spreads in luminosities of members of 
Table 1

Approximate Uncertainties in Stellar Properties

\begin{tabular}{lcl}
\hline \hline Parameter & $T_{\text {eff }}$ Range & Uncertainty \\
\hline Temperature & $>4100 \mathrm{~K}$ & $200 \mathrm{~K}$ or 2 SpT subclasses \\
Temperature & $3750-4100 \mathrm{~K}$ & $125 \mathrm{~K}$ or $1 \mathrm{SpT}$ subclass \\
Temperature & $2800-3750 \mathrm{~K}$ & $75 \mathrm{~K}$ or $0.5 \mathrm{SpT}$ subclasses \\
\hline Luminosity & All & $\sim 5 \%$ parallax distance \\
Luminosity & All & $10 \%-20 \%$ kinematic distance \\
Luminosity & All & $2 \%-5 \%$ bolometric correction \\
\hline
\end{tabular}

Note.

${ }^{\mathrm{a}}$ Or $0.02-0.05$ in mag.

young clusters have been interpreted as true age spreads. For example, in the Orion Nebula Cluster (ONC) a $1.5 \mathrm{dex}$ luminosity spread (da Rio et al. 2010) led Reggiani et al. (2011) to claim an age spread of 1.5-3.5 Myr, although some of this spread may alternately be interpreted as a spread in radius from different accretion histories (Hartmann et al. 1997; Baraffe et al. 2009). Improved spectral types and extinction measurements of two outliers in the ONC sample indicate that the luminosity spread may be overestimated (Manara et al. 2013). If we consider $\sim 2 \mathrm{Myr}$ as an approximate age spread, then that would lead to $0.16 \mathrm{dex}$ and 0.08 dex spreads in luminosities of $5 \mathrm{Myr}$ old and $20 \mathrm{Myr}$ old clusters of single stars (as assessed for $3500 \mathrm{~K}$ stars from the Baraffe tracks; see Section 2.5). These luminosity spreads are consistent with the $0.05-0.15$ dex errors in placing young stars in the HR diagram (see Table 1; Preibisch 2012; Soderblom et al. 2014).

In this paper, we use literature spectral types and photometry to construct empirical isochrones of luminosity versus temperature of diskless low mass stars in nearby associations and compare them to pre-main-sequence isochrones. This approach is similar to the empirical isochrones derived by Hillenbrand (2009) and by Bell et al. (2012, 2013, 2014), but is focused exclusively on low mass stars in nearby young associations and moving groups and is motivated by a desire for deriving simple formulations that approximate empirical isochrones in HR diagrams. In Section 2, we describe the literature data that are used to place stars on HR diagrams and the pre-main-sequence evolutionary tracks used for age estimates. In Section 3 we develop empirical isochrones for a set of benchmark young associations. In Section 4, the empirical isochrones are compared to isochrones predicted from models of pre-main-sequence evolution to test evolutionary tracks and to obtain age estimates for the young clusters for each model. In Section 5, we discuss how the ages from different tracks compare with independent age measurements.

\section{SAMPLE, DATA, AND PRE-MAIN-SEQUENCE EVOLUTION MODELS}

\subsection{Membership and Properties of Nearby Moving Groups}

The $\beta$ Pic and Tuc-Hor Moving Groups and the $\epsilon$ Cha, $\eta$ Cha, and TW Hya Associations are used in this paper as benchmark clusters to measure empirical isochrones of young regions. The results obtained from these benchmark clusters are then applied to the Upper Sco OB Association. Searches for membership in existing all sky surveys, along with analyses of proper motions and radial velocities, have substantially improved the confidence in membership for these associations.
Meanwhile, the proliferation of accurate parallax measurements for the nearest groups has significantly reduced the uncertainty in luminosities arising from errors in distance measurements. When combined, these advances allow for a robust analysis of the stellar loci of these clusters.

In some analysis below, the two older associations (the $\beta$ Pic and Tuc-Hor Moving Groups) and the three younger associations (TW Hya, $\eta$ Cha, and $\epsilon$ Cha) are grouped together. Gravity differences between these clusters are described in Sections 2.2 and 2.3, and the similarity in ages among the older and younger groups will be established in Section 3. The binary census is discussed in this section, while the implementation of binaries is described in Section 2.4.

Beta Pic Moving Group. Membership for the $\beta$ Pic moving group is obtained from the analysis of Malo et al. $(2013,2014)$, based on a $>90 \%$ probability calculated from proper motion, radial velocity, photometry, and parallaxes when available. Spectral types are obtained from their compilation of literature spectral types. Members with distances obtained from kinematic modeling are not used in this paper because many targets are close enough that the percentage uncertainties are too large. ${ }^{3}$ Binarity was accounted for based on the compilation of Malo et al. (2013), which relied heavily on Janson et al. (2012) for M dwarfs. A few objects and binary information are added from Riedel et al. (2014).

Tuc-Hor. The membership and spectral types for the TucHor Association are obtained from Kraus et al. (2014). The distances used here are from their kinematic analysis. 4 The spectral types used here are obtained from their spectral analysis rather than the spectral energy distributions. Binary information is not included in our analysis of Tuc-Hor.

$\epsilon$ Cha Association. Membership, spectral types, and kinematic distances of $\epsilon$ Cha members were obtained from Murphy et al. (2013). This membership list is mostly consistent with but more complete than that of Lopez Marti et al. (2013). The targets listed as members in Lopez Marti et al. (2013) but excluded from Murphy et al. (2013) are VW Cha and CM Cha, both of which have disks and would therefore not be included in our analysis, and 2MASS J12074597-7816064, which was identified by Fang et al. (2013) as a proper motion outlier. The kinematic distances from Murphy et al. (2013) are adopted here and have a median of $111 \mathrm{pc}$. The known binaries are accounted for following the description in Murphy et al. (2013), though the binary census is poor.

$\eta$ Cha Association. Membership in the $\eta$ Cha association is based primarily on the initial identification by Mamajek et al. (1999) and on a follow-up census by Luhman \& Steeghs (2004). The spectral types for those initial 18 members are obtained from Luhman \& Steeghs (2004). Five dispersed very low mass stars were found and characterized by Murphy et al. (2010) and are also included here. The additional members found by Lopez Marti et al. (2013) based on proper motions

\footnotetext{
3 The confirmed members ( $>90 \%$ probability) with parallax distances occupy a tight stellar locus, with a luminosity scatter of 0.14 dex around the best fit line. Confirmed members between 3300 and $5000 \mathrm{~K}$ with only kinematic distances have a luminosity scatter (standard deviation about the mean linear fit) of $0.36 \mathrm{dex}(0.25 \mathrm{dex}$ if restricted to $3350-5000 \mathrm{~K})$.

4 Unlike the case for the $\beta$ Pic Moving Group, the kinematic distances for Tuc-Hor produce a tight stellar locus, perhaps because the average distance is larger so the fractional uncertainty is smaller. Kraus et al. (2014) calculate that a $5 \%$ uncertainty in kinematic distance measurements applies to stars in their

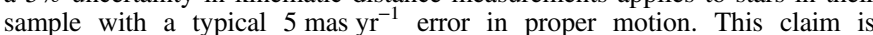
supported by parallax measurements of five stars (van Leeuwen 2007; Riedel et al. 2014) that differ by $4 \%$ from the kinematic distances in Kraus et al.
} 

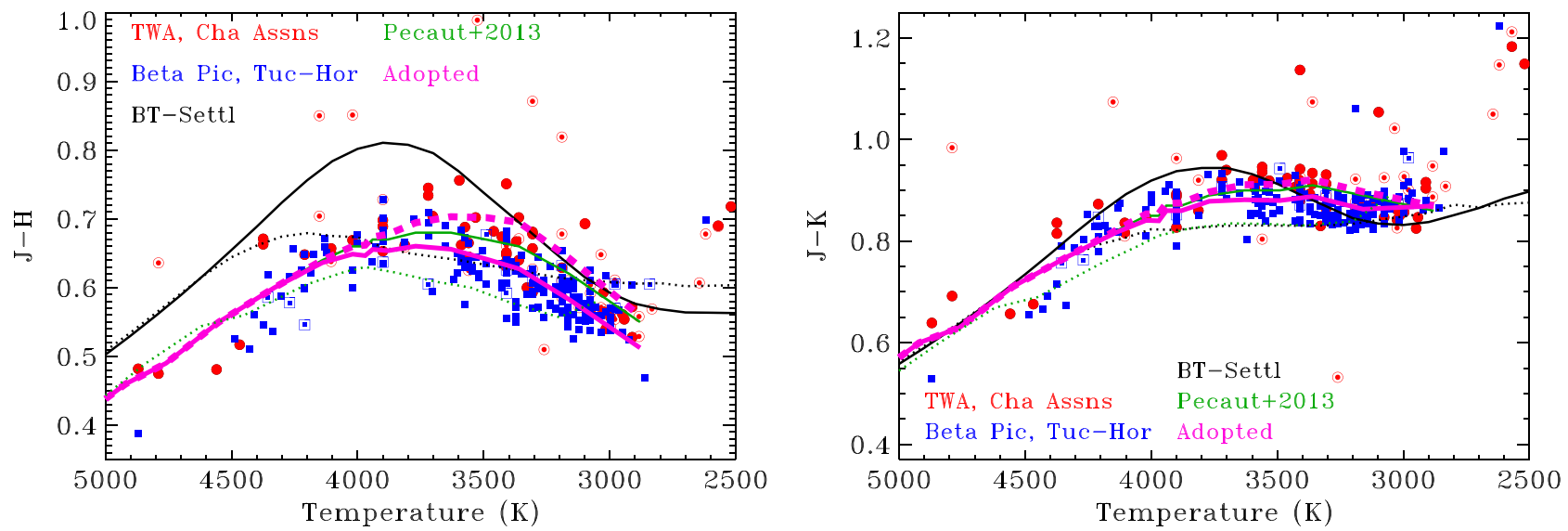

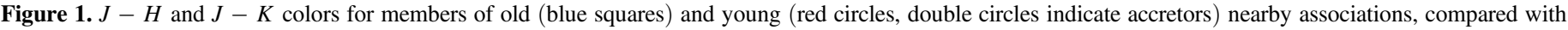

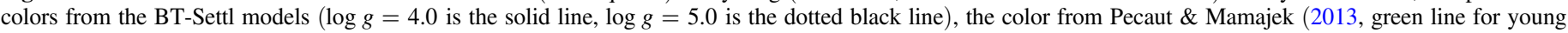

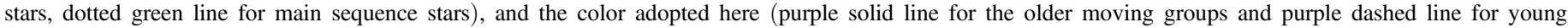

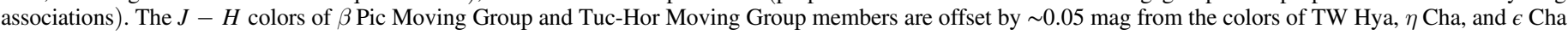

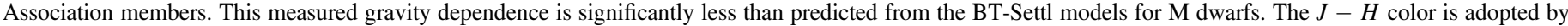
approximating the $J-H$ color intermediate between the younger and older clusters.

and the Covino et al. (1997) X-ray survey are excluded here because they are far from the cluster core and may therefore be located at a different distance. The distance to all members is assumed to be $94 \mathrm{pc}$, the average Hipparcos parallax distance of $\eta$ Cha and RS Cha (van Leeuwen 2007; see discussion in Murphy et al. 2010). An accounting of spatially resolved binaries was obtained by Brandeker et al. (2006) and is applied here.

TW Hya Association. Membership in the TWA is based on kinematic analyses (Malo et al. 2013, 2014; Weinberger et al. 2013; Ducourant et al. 2014; Riedel et al. 2014). Spectral types of most members and binarity are obtained from the compilation in Herczeg \& Hillenbrand (2014). The new equal luminosity binary TWA member SCR $1012-3124 \mathrm{AB}$ is also included here (Riedel et al. 2014). Although parallax distances are preferred, TWA stars with kinematic distance measurements are included in our analyses. For TWA $8 \mathrm{~A}$ and $8 \mathrm{~B}$, a parallax distance distance of $44 \mathrm{pc}$ is adopted here as the average of parallax distances measured by Ducourant et al. (2014) and Riedel et al. (2014). Three members of the HD 98800 quadruple system are included here with data from Laskar et al. (2009), despite the presence of weak IR emission from HD $98800 \mathrm{~N}$.

Upper Sco OB Association. The stellar census and spectral types for the Upper Sco OB Association are obtained from the compilation of members by Luhman \& Mamajek (2012). Multiplicity is corrected based on several incomplete companion searches (Kraus et al. 2008; Biller et al. 2011; Kraus \& Hillenbrand 2012; Lafreniere et al. 2014). The luminosities estimated for the Upper Sco OB Association members are corrected for extinction (see Section 2.4). The average extinction $^{5}$ of $A_{V}=0.46$ mag for low mass stars and $0.58 \mathrm{mag}$ for brown dwarfs is intermediate between the average extinction of $A_{V}=0.78 \mathrm{mag}$ for A stars, $A_{V}=0.59$ mag for G stars, and $A_{V}=0.35$ mag for F stars Pecaut et al. (2012). Stars with $A_{V}>2$ mag are atypical for Upper Sco and are not included in the HR diagram analysis. All stars in Upper

\footnotetext{
5 The extinction for both low mass stars and brown dwarfs is 0.66 mag if the photospheric $J-K$ color is adopted from the older moving groups rather than the younger clusters.
}

Sco are assumed to be located at a distance of $145 \mathrm{pc}$ (de Zeeuw et al. 1999). The cluster is $30 \mathrm{pc}$ wide, and a depth of similar length likely contributes to the large luminosity spread that has been measured for Upper Sco (Slesnick et al. 2008).

Stars without any WISE W2 or W3 photometry (2 of 214 total stars between 3200 and $5000 \mathrm{~K} ; 7$ of 415 total brown dwarfs between 2800 and $3200 \mathrm{~K}$ ) are included in the Upper Sco sample because their exclusion could bias any results at low masses against faint objects.

\subsection{Colors of The Five Benchmark Clusters}

Photometry for members of the five benchmark clusters is obtained from the 2MASS JHKs survey (Skrutskie et al. 2006), WISE W1, W2, W3, W4 survey (Cutri et al. 2012), the UCAC4 APASS all-sky BVgri survey (Zacharias et al. 2013), and the GALEX NUV/FUV survey (Bianchi et al. 2011). This collection of photometry is used in the following subsections to identify and exclude disks, to ensure that extinction is negligible (or correct luminosities for extinction, in the case of Upper Sco members), and to calculate bolometric corrections (see also Appendix). All objects are detected in JHK. Some objects lack photometry at other bands.

Broadband colors of objects in the younger TW Hya, $\eta$ Cha, and $\epsilon$ Cha Associations are mostly consistent with those from the older $\beta$ Pic and Tuc-Hor Moving Groups, which all form a tight stellar locus in infrared and optical colors (Figure 1 and Appendix). The contraction to the main sequence corresponds with a gradual increase in gravity. Just as different gravities between main sequence and young stars yield color differences (e.g., Luhman et al. 2003; Pecaut \& Mamajek 2013), corresponding differences should be present between 5 and $30 \mathrm{Myr}$ old pre-main-sequence stars. The $J-H$ color is redder for the younger associations relative to the older moving groups (Table 2 and right panel of Figure 1), which is the result of gravity differences and possible mismatches between SpT and temperature between the two samples. The $J-K$ color is also $\sim 0.03$ mag redder for early- to mid-M stars in younger associations. Most empirical colors for the sample stars are also consistent with the intrinsic colors for the 5-30 Myr stars as assessed by Pecaut \& Mamajek (2013). 
Table 2

Temperatures and Bolometric Corrections vs. Spectral Type

\begin{tabular}{|c|c|c|c|c|c|c|c|}
\hline \multirow[b]{2}{*}{ SpT } & \multicolumn{2}{|c|}{$T_{\text {eff }}$} & \multicolumn{2}{|c|}{$\mathrm{BC}_{\mathrm{J}}^{\mathrm{a}}$} & \multicolumn{3}{|c|}{$J-H$} \\
\hline & $\mathrm{HH} 14^{\mathrm{b}}$ & $\mathrm{PM} 13^{\mathrm{c}}$ & Here & $\mathrm{PM} 13^{\mathrm{c}}$ & Old $^{\mathrm{d}}$ & Young $^{\mathrm{d}}$ & PM13 \\
\hline F5 & 6600 & 6420 & 0.79 & 0.85 & 0.17 & 0.17 & 0.19 \\
\hline F8 & 6130 & 6100 & 0.95 & 0.96 & 0.22 & 0.22 & 0.23 \\
\hline G0 & 5930 & 6050 & 1.02 & 0.98 & 0.26 & 0.26 & 0.24 \\
\hline $\mathrm{G} 2$ & 5690 & 5870 & 1.10 & 1.03 & 0.30 & 0.30 & 0.27 \\
\hline G5 & 5430 & 5500 & 1.18 & 1.16 & 0.34 & 0.34 & 0.33 \\
\hline G8 & 5180 & 5210 & 1.26 & 1.25 & 0.40 & 0.40 & 0.39 \\
\hline $\mathrm{K} 0$ & 4870 & 5030 & 1.36 & 1.30 & 0.47 & 0.47 & 0.43 \\
\hline $\mathrm{K} 2$ & 4710 & 4760 & 1.41 & 1.40 & 0.50 & 0.50 & 0.49 \\
\hline K5 & 4210 & 4140 & 1.56 & 1.58 & 0.63 & 0.63 & 0.64 \\
\hline K7 & 4020 & 3970 & 1.62 & 1.63 & 0.66 & 0.66 & 0.66 \\
\hline M0 & 3900 & 3770 & 1.66 & 1.69 & 0.67 & 0.68 & 0.68 \\
\hline M1 & 3720 & 3630 & 1.73 & 1.74 & 0.67 & 0.70 & 0.68 \\
\hline M2 & 3560 & 3490 & 1.78 & 1.80 & 0.65 & 0.70 & 0.67 \\
\hline M3 & 3410 & 3360 & 1.84 & 1.84 & 0.63 & 0.70 & 0.66 \\
\hline M4 & 3190 & 3160 & 1.93 & 1.91 & 0.58 & 0.65 & 0.62 \\
\hline M5 & 2980 & 2880 & 1.99 & 2.01 & 0.53 & 0.59 & 0.55 \\
\hline M6 & 2860 & $\cdots$ & 2.03 & $\cdots$ & 0.50 & 0.55 & $\cdots$ \\
\hline M7 & 2770 & $\cdots$ & 2.06 & $\cdots$ & 0.48 & 0.53 & $\cdots$ \\
\hline
\end{tabular}

Note.

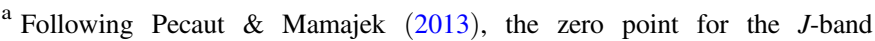
magnitude is $3.129 \times 10^{-10} \mathrm{erg} \mathrm{cm}^{-2} \mathrm{~s}^{-1} \AA^{-1}$ and for the absolute magnitude is $3.055 \times 10^{35} \mathrm{erg} \mathrm{cm}^{-2} \mathrm{~s}^{-1}$.

${ }^{\mathrm{b}}$ HH14 refers to Herczeg \& Hillenbrand (2014).

${ }^{c}$ PM13 refers to the 5-30 Myr stars in Table 6 of Pecaut \& Mamajek (2013).

d $J-H$ colors given for the median of diskless stars from the two old moving groups and three young associations.

The sample is restricted to diskless stars. Disks are identified by at least one of a $K-\mathrm{W} 3$ excess or $K-\mathrm{W} 2$ of 0.5 mag above the photospheric level, as determined from the observed stellar locus and from photospheric models (Figure 2; see also, e.g., Luhman \& Mamajek 2012). A few stars may retain transition or debris disks that do not appear as a $K-\mathrm{W} 2$ or $K-\mathrm{W} 3$ excess, but these disks are unlikely to affect the $J$-band brightness of the star. The sources 2MASS J08014860-8058052, 2MASS J11550485-7919108, and 2MASS J02153328-5627175 fall below our disk criteria but are excluded in our analysis because of excess emission in the WISE W4 band or the detection of accretion in optical spectra (Murphy et al. 2011, 2013; Cutri et al. 2012).

\subsection{Bolometric Corrections and Temperature Scale}

The luminosities of stars in the five young, benchmark associations are calculated by applying bolometric corrections to 2MASS $J$-band magnitudes (Skrutskie et al. 2006). Any bulk offset between the actual and implemented bolometric correction would lead to an offset in luminosity and therefore age, while any temperature dependence in bolometric correction offset would change the relative luminosities/ages of stars in the same association but with different temperatures. These systematic uncertainties introduce problems when comparing different methods and when comparing observations to models. In this subsection, we calculate bolometric corrections and subsequently assess temperature dependence in the uncertainties by comparing results from temperature and bolometric corrections derived here to those of Pecaut \& Mamajek (2013). The Appendix describes the color corrections and constants that are used here to calculate fluxes and stellar luminosities.
Our bolometric corrections are calculated from the BT-Settl model atmospheres (version CIFITS2011bc, which use abundances from Caffau et al. 2011) with $\log g=4.0$ (Allard et al. 2012). Minor offsets relative to the $J$-band flux are applied to several spectral bands to account for differences between synthetic and observed photometry (see Figure 13). Spectral types are converted to temperature from the conversion scale of Herczeg \& Hillenbrand (2014), which was calculated by comparing low resolution optical blue-red spectra to 2011 BT-Settl models. The model spectra and observed photometry are compared in magnitude space. The synthetic photometry is calculated by convolving the BT-Settl model spectra in photon units with filter transmission curves (see the Appendix). The empirical colors are obtained from the photometry described in Section 2.2. The primary uncertainties in these empirical corrections are introduced by the lack of photometric coverage in the $\mathrm{z}$ and $\mathrm{Y}$ bands and in near-IR regions with poor telluric transmission, which are located between the zYJHK filters.

Table 2 compares our spectral type-temperature conversion and bolometric corrections to the 5-30 Myr stars of Pecaut \& Mamajek (2013). In general, temperatures in Pecaut \& Mamajek (2013) are 100-200 K cooler than those presented here. These errors are roughly assessed from the uncertainty in assigning spectral types from low resolution optical spectra. Pecaut \& Mamajek (2013) also include K8 and K9 spectral types. Their SpT-temperature conversion extend only to M5, so results for brown dwarfs (Section 3.2) are limited to the conversions of Herczeg \& Hillenbrand (2014). When evaluated at the same temperature, the bolometric corrections measured directly from the BT-Settl models with the Caffau et al. (2011) abundances reproduce the Pecaut \& Mamajek (2013) bolometric corrections to within $1 \%$ at all spectral types, even though Pecaut \& Mamajek (2013) used models with abundances from Asplund et al. (2009). Differences between our bolometric corrections and those of Pecaut et al. are introduced by differences in the SpT-temperature conversion and by our tweaking of the BT-Settl models to reproduce the measured colors. The bolometric correction versus spectral type is also different because of the different spectral type-temperature conversion scale. As a consequence, when compared by temperature, bolometric corrections are similar for $\mathrm{M}$ dwarfs and $2 \%$ smaller here for $\mathrm{K}$ dwarfs. The practical result of these differences is that temperatures are $\sim 50-150 \mathrm{~K}$ higher and luminosities are $\sim 1.5 \%-4.5 \%$ brighter relative to those calculated from Pecaut \& Mamajek (2013) bolometric correction and temperature scales.

The analysis of stellar temperatures and luminosities for 3200-5000 K stars (Section 3.1) is repeated for the Pecaut \& Mamajek (2013) SpT-temperature conversions and bolometric corrections. Both Pecaut \& Mamajek (2013) and Herczeg \& Hillenbrand (2014) base their SpT-temperature conversions on the BT-Settl models, although Tottle \& Mohanty (2015) find discrepancies between these models and near-IR colors at both early- and late-M spectral types. While the SpT-temperature conversion is still a significant source of uncertainty, the main results of this paper are robust to systematic uncertainties in these scales.

\subsection{HR Diagram Methodology}

Stellar luminosities are calculated from 2MASS J-band brightness, literature spectral types, and stellar distances 


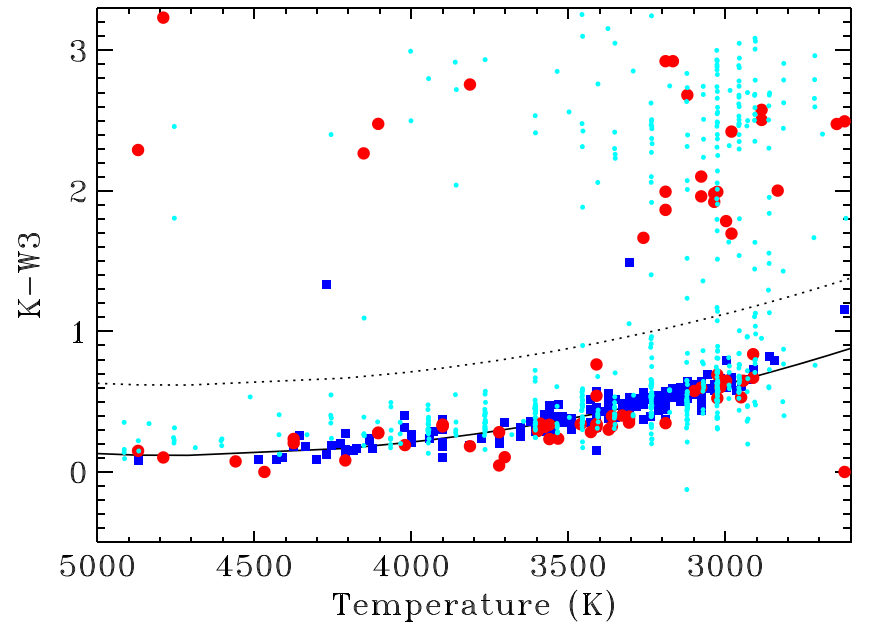

Figure 2. K-W3 color as a disk indicator. The samples analyzed here are restricted to diskless stars, identified by a K-W3 color less than 0.5 mag above the best fit second order polynomial (solid black line shows photospheric K-W3 color; dotted black line shows the cutoff for the presence of excess W3 emission). The red circles are stars in the three younger associations, the blue squares are stars in the two Moving Groups, and the small cyan circles are stars in Upper Sco.

calculated from parallax measurements or from association kinematics. The $J$ band is used for luminosity calculations because of the uniform reliability of the 2MASS survey, and because the $J$ band is near the peak of the spectral energy distribution for late type stars, which minimizes the uncertainty in bolometric correction caused by spectral type errors. The stars are diskless, so accretion and disk extinction do not affect the observed $J$ band photometry. Stars in each association are assumed to be co-eval.

Table 1 lists the uncertainties applied here for luminosity and temperature estimates. Luminosity uncertainties are calculated from an assumed 5\% uncertainty from a combination of photometric and bolometric correction errors and from distance uncertainties, typically $<5 \%$ for parallax distances $(5 \%$ distance uncertainty is assessed for all $\eta$ Cha Association members), $5 \%$ for kinematic distances for Tuc-Hor members (see footnote 3 ), and $10 \%$ for kinematic distances of other regions. Bolometric corrections for the $J$ band are described in detail in the next section and are more uncertain for cooler stars with large opacities in molecules. The depth of Upper Sco adds uncertainty to the distance and therefore luminosity for individual members, which contributes to its luminosity spread. Temperature uncertainties are based on estimated errors and methodological differences in spectral typing (see discussion in Herczeg \& Hillenbrand 2014). Systematic differences in spectral typing between different studies will also affect the HR diagrams constructed in this paper.

In multiple systems, all members with resolved spectral types are included in this analysis. Unresolved spectral types are included if the stars are spatially resolved in $J, H$, or $K$ bands. In these cases, the primary is assumed to have the system spectral type. The $J$ band magnitude of the primary is calculated by assuming that the brightness difference is constant in the near-IR (the $J-H$ and $J-K$ colors are roughly constant for $\mathrm{M}$ dwarfs). Optically resolved or doublelined spectroscopic binaries are included if both stars are of similar brightness. Single-lined binaries are excluded.
Extinction is assumed to be negligible for all diskless stars in the five benchmark associations (see colors in Section 2.2 and the Appendix). Stars in the younger associations have a slightly bluer $g-J$ color, which is the opposite of expectations if extinction were important and supports the assumption that extinction is negligible. The four stars in $\epsilon$ Cha with $A_{V}>1.5$ mag, as calculated by Murphy et al. (2013), all have disks, which may have either produced extinction or may have altered the near-IR colors sufficiently to have led to extinction in their calculations.

However, extinction affects luminosities of stars in the Upper Sco OB Association. A color excess $E(J-K)$ is calculated for each star here by measuring the difference between the observed $J-K$ color the $J-K$ color adopted for the young associations, as shown as the dashed purple line in Figure 1. The extinction $A_{J}$ is then calculated and corrected for by assuming a total-to-selective extinction of $A_{J}=0.282 A_{V}$, and $A_{J}=1.66 E(J-K)$ from Rieke \& Lebofsky (1985). If the total-to-selective extinction $R_{V}=4$ instead of 3.1, the $A_{J}$ values would increase by $0.07 \%$, as calculated from the extinction curves of Weingartner \& Draine (2001).

Young $\mathrm{K}$ and $\mathrm{M}$ stars without disks have extinctions accurate to $\sim 0.2 \mathrm{mag}$, although the precise error in extinction depends on the error in spectral type. Stars with extinctions $A_{V}>2$ mag are excluded from our analysis. Negative values for extinction are retained and applied for statistical purposes.

\subsection{Models of Pre-main-sequence Evolution}

The empirical isochrones derived in Section 3 are compared to model pre-main-sequence isochrones in Section 4. Stassun et al. (2014) summarize currently available pre-main-sequence evolutionary tracks. The evolutionary models used here, D'Antona \& Mazzitelli (1997, hereafter DM97), Siess et al. (2000, hereafter Siess), Baraffe et al. (1998, (BCAH98), 2003), Dotter et al. (2008, hereafter Dartmouth), Tognelli et al. (2011, hereafter Pisa), and PARSECv1.2 s (Bressan et al. 2012, Chen et al. 2014; herafter PARSEC, also frequently referred to as Padova), are selected based on their availability and prevalence in the pre-main-sequence literature. The BCAH98 models have mixing lengths $\alpha=1.0$ (BCAH98) or mixing length $\alpha=1.9$ (hereafter BCAH98-1.9). The PARSEC models are selected for their artificial tweaks to the $T-\tau$ relationship which reproduce the color-magnitude diagrams of very low mass stars in Praesepe and M67. For stars below $4000 \mathrm{~K}$, stars of the same age and mass are much cooler and are only slightly less luminous (because of larger radii) in the PARSECv1.2 s models relative to the PARSECv1.1 models (Bressan et al. 2012). The PARSECv1.1 models are used only in Table 3 and are not discussed further.

In addition to these sets of models, we include analysis of newly available tracks calculated by Baraffe et al. (2015, hereafter BHAC-2015) using the BT-Settl atmospheric models and a prescription of convection based on 2D/3D models, which is an improvement from the mixing length prescription for convection. We also include new Dartmouth tracks, which improve the thermal structure in the atmosphere by calculating fitting to large optical depths (G. A. Feiden et al. 2015, in preparation; G. A. Feiden et al. 2015, private communication, hereafter Feiden). The Feiden models use the Grevesse \& Sauval (1998) abundances with PHOENIX AMES-COND atmospheres (Hauschildt et al. 1999a, 1999b). Stassun et al. (2014) include preliminary descriptions of the Feiden tracks, 
Table 3

Slopes of Linear Fits to Young Stellar Isochrones

\begin{tabular}{|c|c|c|c|c|c|}
\hline \multirow{2}{*}{\multicolumn{2}{|c|}{ Empirical Fit }} & \multicolumn{2}{|c|}{$3200-5000 \mathrm{~K}$} & \multicolumn{2}{|c|}{$2800-3200 \mathrm{~K}$} \\
\hline & & Slope & StD & Slope & StD \\
\hline \multicolumn{2}{|l|}{ Combined } & $0.76 \pm 0.07$ & 0.15 & $2.05 \pm 0.15$ & 0.18 \\
\hline \multirow{2}{*}{\multicolumn{2}{|c|}{$\begin{array}{l}\text { Young Groups Only } \\
\text { Old Groups Only }\end{array}$}} & $0.76 \pm 0.06$ & 0.16 & $3.1 \pm 0.8$ & 0.20 \\
\hline & & $0.85 \pm 0.08$ & 0.14 & $2.05 \pm 0.18$ & 0.18 \\
\hline \multirow{5}{*}{\multicolumn{2}{|c|}{$\begin{array}{l}\text { Tuc Hor } \\
\text { BPMG } \\
\text { TWA } \\
\epsilon \text { Cha } \\
\eta \text { Cha }\end{array}$}} & $0.83 \pm 0.12$ & 0.14 & $2.28 \pm 0.25$ & 0.18 \\
\hline & & $0.91 \pm 0.12$ & 0.14 & $1.87 \pm 0.38$ & 0.16 \\
\hline & & $0.82 \pm 0.15$ & 0.17 & d & $\ldots$ \\
\hline & & $0.73 \pm 0.08$ & 0.17 & d & $\cdots$ \\
\hline & & $0.93 \pm 0.27$ & 0.06 & d & $\ldots$ \\
\hline \multicolumn{2}{|l|}{ Upper Sco } & $0.85 \pm 0.07$ & 0.18 & $3.5 \pm 0.8$ & 0.27 \\
\hline Model & $\mathrm{Age}^{\mathrm{c}}$ & $3400-5000 \mathrm{~K}$ & & $2800-3200 \mathrm{~K}$ & \\
\hline ВСАН98 & 3 & 1.04 & & 1.97 & $\ldots$ \\
\hline BCAH98 & 20 & 0.89 & & 1.62 & $\ldots$ \\
\hline ВСАН98-1.9 & 3 & 0.69 & & $\ldots$ & $\ldots$ \\
\hline ВСАН98-1.9 & 20 & 0.76 & & $\ldots$ & $\cdots$ \\
\hline ВНАC-2015 & 3 & 0.68 & & 1.49 & $\cdots$ \\
\hline BНAC-2015 & 20 & 0.72 & & 1.84 & $\ldots$ \\
\hline Pisa & 3 & 0.71 & & $\cdots$ & $\cdots$ \\
\hline Pisa & 20 & 0.76 & & $\cdots$ & $\cdots$ \\
\hline Dartmouth & 3 & 0.73 & & $\cdots$ & $\cdots$ \\
\hline Dartmouth & 20 & 0.74 & & $\cdots$ & $\cdots$ \\
\hline Feiden & 3 & 0.75 & & 1.57 & $\cdots$ \\
\hline Feiden & 20 & 0.84 & & 2.02 & $\ldots$ \\
\hline Siess & 3 & 0.87 & & $\ldots$ & $\ldots$ \\
\hline Siess & 20 & 1.00 & & $\cdots$ & $\cdots$ \\
\hline DM97 & 3 & 0.70 & & 2.46 & $\ldots$ \\
\hline DM97 & 20 & 0.85 & & 2.94 & $\cdots$ \\
\hline PARSEC & 3 & 0.62 & & 1.15 & $\cdots$ \\
\hline PARSEC & 20 & 0.51 & & 1.32 & $\ldots$ \\
\hline PARSECv1.1 & 3 & 0.83 & & $\cdots$ & $\cdots$ \\
\hline PARSECv1.1 & 20 & 1.09 & & $\cdots$ & $\cdots$ \\
\hline
\end{tabular}

Notes. Slope (in $\log L$ per $10^{3} \mathrm{~K}$ ), uncertainty, and standard deviation of fits to individual, combined, and model isochrones.

${ }^{\mathrm{a}} \eta \mathrm{Cha}, \epsilon$ Cha, and TW Hya Associations.

b $\beta$ Pic and Tuc Hor Moving Groups.

${ }^{c}$ Age of the model isochrone in Myr.

${ }^{\mathrm{d}}$ Insufficient number of data points.

while Malo et al. (2014) use a magnetic version of the Feiden tracks. The BHAC-2015 and Feiden models predict very similar temperature/luminosity evolution for low mass stars and brown dwarfs, which then yield very similar isochrones (Figure 4).

All model tracks use solar abundances, ${ }^{6}$ which is consistent with abundance measurements of young stars in the solar neighborhood (e.g., D'Orazi et al. 2011). Only the BCAH98 and the DM97 pre-main-sequence tracks are available for masses $<0.1 M_{\odot}$, while the Pisa models are only available for masses $>0.2 M_{\odot}$. The BCAH98 models with $\alpha=1.0$ are available for stars less massive than $<1.4 M_{\odot}$ while only models between 0.6 and $1.4 M_{\odot}$ have been calculated with $\alpha=1.9$. The PARSEC models are only calculated to $0.1 M_{\odot}$, but extend to low temperatures $(2800 \mathrm{~K}$ at $1 \mathrm{Myr}, 2500 \mathrm{~K}$ at $10 \mathrm{Myr}$ ) because the tweaks to the $T-\tau$ relationship reduce the effective temperature of very low mass stars.

\footnotetext{
6 Changes in solar abundance measurements mean that these models may use different abundances and are therefore not directly comparable.
}

Table 4

Luminosity Ordering of Young Clusters

\begin{tabular}{lcccccccc}
\hline \hline Stellar & & \multicolumn{3}{c}{ Low Mass Stars $^{\mathrm{a}}$} & & \multicolumn{3}{c}{ Brown Dwarfs $^{\mathrm{b}}$} \\
\cline { 3 - 5 } \cline { 7 - 9 } Association & $d^{\mathrm{c}}$ & $N$ & $\log L_{4200}$ & $\mathrm{StD}$ & & $N$ & $\log L_{3000}$ & $\mathrm{StD}$ \\
\hline Upper Sco & 145 & 176 & -0.25 & 0.18 & & 286 & -1.51 & 0.27 \\
$\epsilon$ Cha & 111 & 14 & -0.28 & 0.17 & & 4 & -1.42 & 0.23 \\
$\eta$ Cha & 94 & 7 & -0.32 & 0.06 & & 5 & -1.61 & 0.16 \\
TW Hya & $(57)$ & 22 & -0.40 & 0.17 & & 3 & -1.51 & 0.22 \\
$\beta$ Pic & $(30)$ & 16 & -0.65 & 0.14 & & 11 & -1.91 & 0.15 \\
Tuc Hor & $(51)$ & 48 & -0.72 & 0.14 & & 89 & -2.20 & 0.18 \\
\hline
\end{tabular}

Notes.

${ }^{\text {a }}$ From linear fits to $3200 / 3400-5000 \mathrm{~K}$ stars; see Section 3.1.

${ }^{\mathrm{b}}$ From temperture shifts for stars of 2800-3200 K; see Section 3.2.

${ }^{\mathrm{c}}$ Distances in () are median association distances.

Figure 3 shows the conversion to age at fixed luminosity and at fixed temperature for the different pre-main sequence evolutionary models. The DM97 model tracks are significant outliers (see also, e.g., Hillenbrand 1997; Bell et al. 2012; Andrews et al. 2013). Their photospheric temperatures are hotter for stars of the same mass, so the corresponding mass of $\mathrm{K}$ and $\mathrm{M}$ stars is much lower than obtained from other evolutionary tracks. The measured luminosity for a star of that mass is therefore high, leading to a young age. At ages $<3 \mathrm{Myr}$, the Dartmouth tracks also yield ages that are younger than the other tracks. In contrast, the BCAH98 tracks assessed for $4200 \mathrm{~K}$ stars are outliers in yielding older ages.

All models predict similar luminosities for stars of the same mass for ages older than $2 \mathrm{Myr}$, despite different temperatures (Figure 5). With accurate age dating of young clusters, masses may be inferred from the luminosity alone and with consistent results between different model tracks.

\section{EMPIRICAL ISOCHRONES FOR THE NEARBY YOUNG MOVING GROUPS}

With an accurate theory of pre-main-sequence evolution, the estimated luminosity of a star at a given estimated temperature could be directly converted to an age. However, scatter in $L$ and $T_{\text {eff }}$ among young cluster members provokes concerns regarding the reliability of age estimates for single stars. Moreover, differences in the slope between observed and model isochrones lead to ages that depend on spectral type. Our approach here focuses on comparing the empirical stellar locus of different associations and interpreting the offsets as relative age differences. Using the stellar loci constructed for young associations, we first focus on empirical trends in $\log L$ versus $T_{\text {eff }}$ (this section) before conducting detailed comparisons to models (Section 4).

Figure 6 shows the combined HR diagrams for the five benchmark young associations. The stellar locus between $3500-5000 \mathrm{~K}$ has a similar shape for all clusters, with offsets in luminosity and temperature. Stars in the older associations have systematically fainter luminosities and warmer temperatures relative to young associations. The slope of the locus is steeper for very low mass stars and brown dwarfs ${ }^{7}(T<3200 \mathrm{~K}$ for the young clusters and $<3400 \mathrm{~K}$ for older clusters) relative to

\footnotetext{
7 These objects include very low mass stars and brown dwarfs and are termed brown dwarfs in this paper for ease of terminology. Object masses are not calculated in this paper, and the distinction between stars and brown dwarfs is not relevant at young ages.
} 

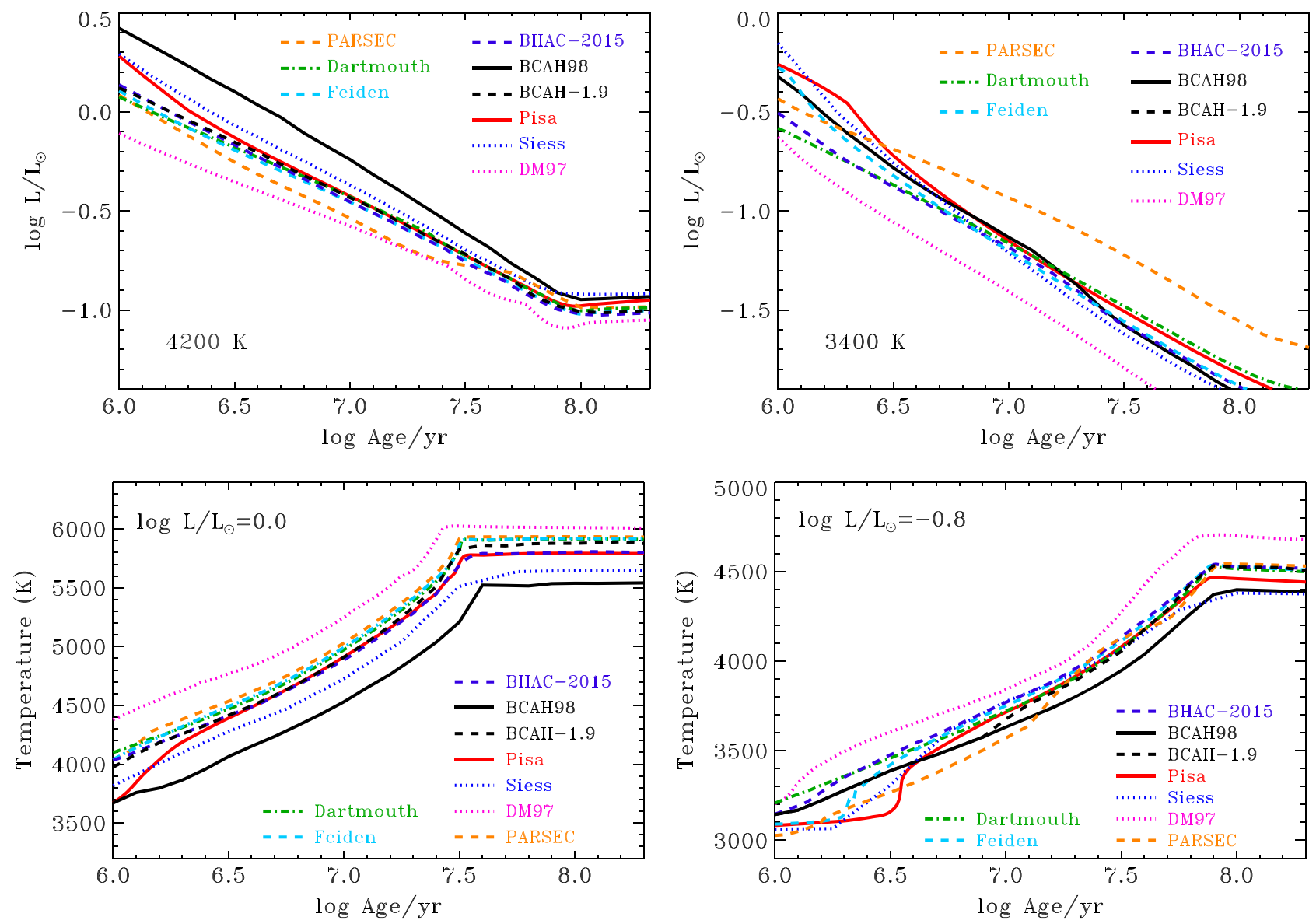

Figure 3. Predictions from different pre-main-sequence evolutionary tracks. The top panels show how luminosity is converted to age for $4200 \mathrm{~K}$ stars (roughly K5 spectral type; left panel) or $3400 \mathrm{~K}$ stars (roughly M3 spectral type; right panel). The bottom panels illustrate the temperature predictions at fixed luminosity. The effective temperatures are much higher in the DM97 models and, for $L=L_{\odot}$, lower in the BCAH98 models.

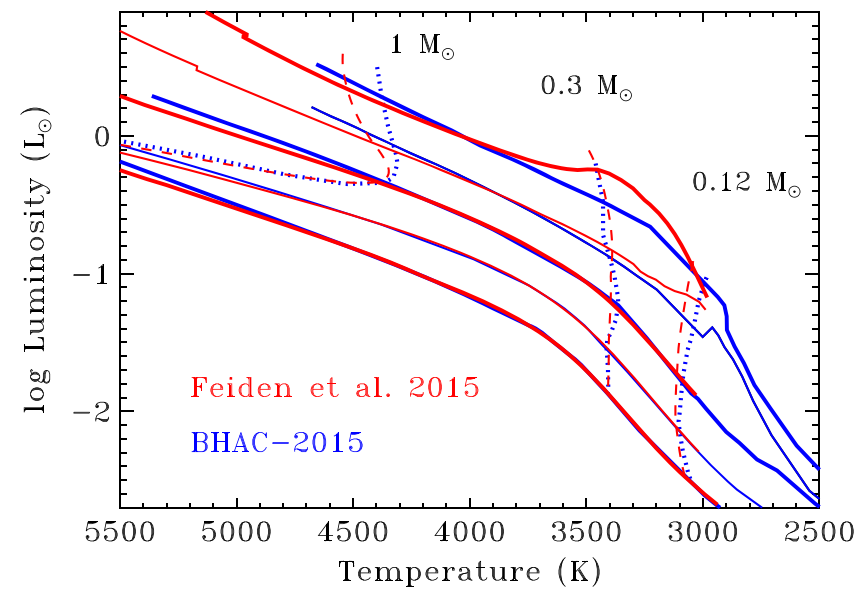

Figure 4. Comparison of the new Feiden (red) and BHAC-2015 (blue) tracks with an improved treatment of convection. The solid lines show isochrones at $\log$ age $\mathrm{yr}^{-1}=6.0,6.5,7.0,7.5$, and 8.0, with 6.0, 7.0, and 8.0 in bold. The vertical lines show the early evolution of $0.12,0.3$, and $1.0 M_{\odot}$ stars from $\log$ age $\mathrm{yr}^{-1}=5.7-8.0$. Although the physics implemented into these modern tracks are different, they produce evolutionary tracks and isochrones that are extraordinarily similar.

the locus for solar mass stars. The location where the break in slope of the temperature-luminosity isochrone occurs is referred to here as the inflection point in the isochrone.
The observed HR diagrams are consistent with the basic shape predicted from isochrones obtained from pre-main-sequence evolutionary models (see comparisons in Section 4 and Figure 11). The gradual decrease in luminosity from 5000 to $\sim 3300 \mathrm{~K}$ is roughly consistent with all pre-main-sequence evolutionary models. At temperatures cooler than $\sim 3300 \mathrm{~K}$, the steep dropoff in luminosity is also roughly consistent with predictions from tracks that calculate pre-main-sequence evolution for stars $<0.2 M_{\odot}$. Based on the HR diagrams, the inflection point where the slope steepens occurs at $\sim 3400 \mathrm{~K}$ for the older two associations and $\sim 3200 \mathrm{~K}$ for younger associations. The temperature intervals are therefore different in fits to stellar and brown dwarf loci of the older and younger associations.

The similarity in stellar loci suggests that the members may be analyzed together after accounting for age-dependent differences between the clusters. In the following subsections, we combine the loci from different associations into a single stellar locus to calculate an empirical isochrone, which can then be generically applied to any young cluster. Combining data from different clusters yields results that are more robust than those calculated for individual, sparsely populated associations. When comparing cluster ages, scatter in data points and different spectral type distributions of members can introduce systematic errors in relative ages.

Once a generalized fit to temperature and luminosity is obtained, scaling the slopes to each cluster in the luminosity direction then provide self-consistent comparisons of relative 

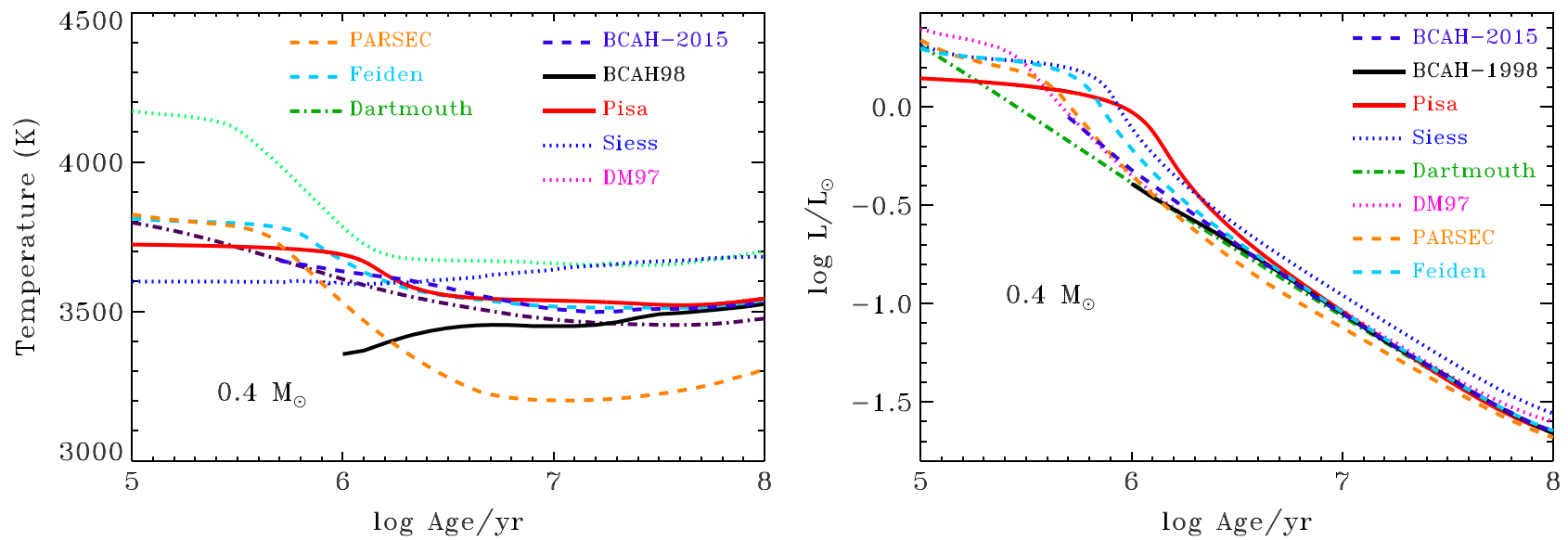

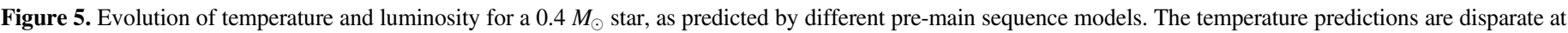

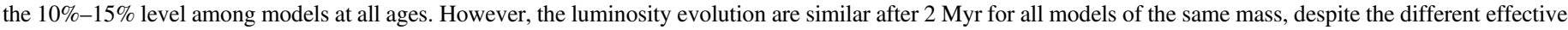
temperatures.

ages that furthermore are robust to certain types of scatter in the temperature-luminosity data points. For example, linear fits to the TWA and Tuc-Hor populations yield median luminosities of $\log L / L_{\odot}=-0.34$ and -0.70 , respectively, for $4200 \mathrm{~K}$ stars, or -1.00 and -1.34 for $3400 \mathrm{~K}$ stars. ${ }^{8}$ These luminosities can be compared among clusters to infer relative ages in $\log L$ as a function of temperature.

The loci of stars above and below the inflection point (3200 K for young regions, $3400 \mathrm{~K}$ for the older regions) are discussed separately in Sections 3.1 and 3.2. An underlying assumption to this approach is that the slopes of stellar loci are constant longward and shortward of the inflection point and that the slopes do not change with cluster age. These sections include a discussion of whether this simplifying assumption is correct. For brown dwarfs in particular, the $\log L-T$ slopes are different for well-populated loci of Upper Sco and Tuc-Hor. The location of the inflection point ranges from $3000-3500 \mathrm{~K}$ and is discussed in Section 3.3.

\subsection{Empirical Isochrones for Low Mass Stars}

In this subsection, we describe global fits to the $3200-5000 \mathrm{~K}$ region $(3400-5000 \mathrm{~K}$ for the $\beta$ Pic and Tuc-Hor Moving Groups), which corresponds to M3-K0 spectral types. Since the empirical loci in this temperature range are similar, a global linear fit to the entire data set provides a consistent assessment of the relative luminosity evolution of a given cluster. In fits to the combined HR diagram of the five benchmark associations, the free parameters are the slope of the line and a different $y$-intercept for each cluster. For illustrative purposes, the different $y$ intercepts are described here as luminosity offsets.

The lower left panel of Figure 6 shows the HR diagram, with each association shifted by the respective difference in $y$ intercept to match the location of the TWA. The empirical isochrone for the combined HR diagram of nearby associations has a best fit slope of

$$
\log L / L_{\odot} \propto(0.76 \pm 0.07) T / 10^{3} \mathrm{~K}\left(T_{\mathrm{infl}}-5000 K\right),
$$

\footnotetext{
8 These luminosities differ from the final values listed in Table 4 because these values are calculated from best-fit slopes for the two clusters, while the final values are obtained from scaling a line with a slope that best fits all regions together.
}

based on linear regressions that includes errors in $T$ and $L$ (as described in Section 2.3) and calculated using mpfitexy.pro in IDL (Williams et al. 2010). The inflection point $T_{\text {infl }}$ used for the fits here is $3200 \mathrm{~K}$ for the younger groups and $3400 \mathrm{~K}$ for the older groups. The $0.07 \mathrm{dex}$ uncertainty includes a $0.05 \mathrm{dex}$ statistical uncertainty and a $\sim 0.05$ dex uncertainty based on different linear fitting techniques, how the data points are weighted, and the inclusion or exclusion of deviant data points. This uncertainty does not include possible errors in the spectral type-temperature conversion or in bolometric corrections, which may depend on spectral type. The standard deviation in luminosity between the observations and best linear fits is $\sim 0.15 \mathrm{dex}$.

The same analysis calculated from the Pecaut \& Mamajek (2013) spectral type-temperature conversion and bolometric corrections produces a slope of $0.72 \pm 0.04 \log L$ per $10^{3} \mathrm{~K}$, which is formally consistent with the results when using the Herczeg \& Hillenbrand (2014) conversions. The different slopes would yield a change in $\log L$ of 0.07 dex between the hottest and coolest stars in the applicable temperature range, which is smaller than other systematic errors.

Table 3 lists the best fit slopes for linear fits to the the combined data set, to individual clusters, and to the clusters combined into the young and old groups. When shifted based on the best fit offsets, the three youngest benchmark associations have steeper slopes than the older clusters, which suggests some evolution with age. The difference in slopes between the young and old clusters, 0.76 and $0.85 \log L$ per $10^{3} \mathrm{~K}$, may lead to systematic differences as large as $0.14 \mathrm{dex}$ for stars at $3400 \mathrm{~K}$ compared with $5000 \mathrm{~K}$.

\subsection{Empirical Isochrones for Young Brown Dwarfs}

In this subsection, we describe global fits to objects cooler than the inflection point in the isochrones $(2800-3400 \mathrm{~K}$ for the Tuc-Hor and $\beta$ Pic Moving Groups, 2800-3200 K for the TW Hya, $\eta$ Cha, and $\epsilon$ Cha Associations). For illustrative purposes, the different $y$-intercepts are visualized as temperature offsets so that the loci overlap (lower right panel of Figure 6). This visualization is practical because the inflection point occurs at different temperatures but approximately the same luminosity (to within a few tenths of dex; see also similar behavior in the model isochrones in Figures 4 and 11). 

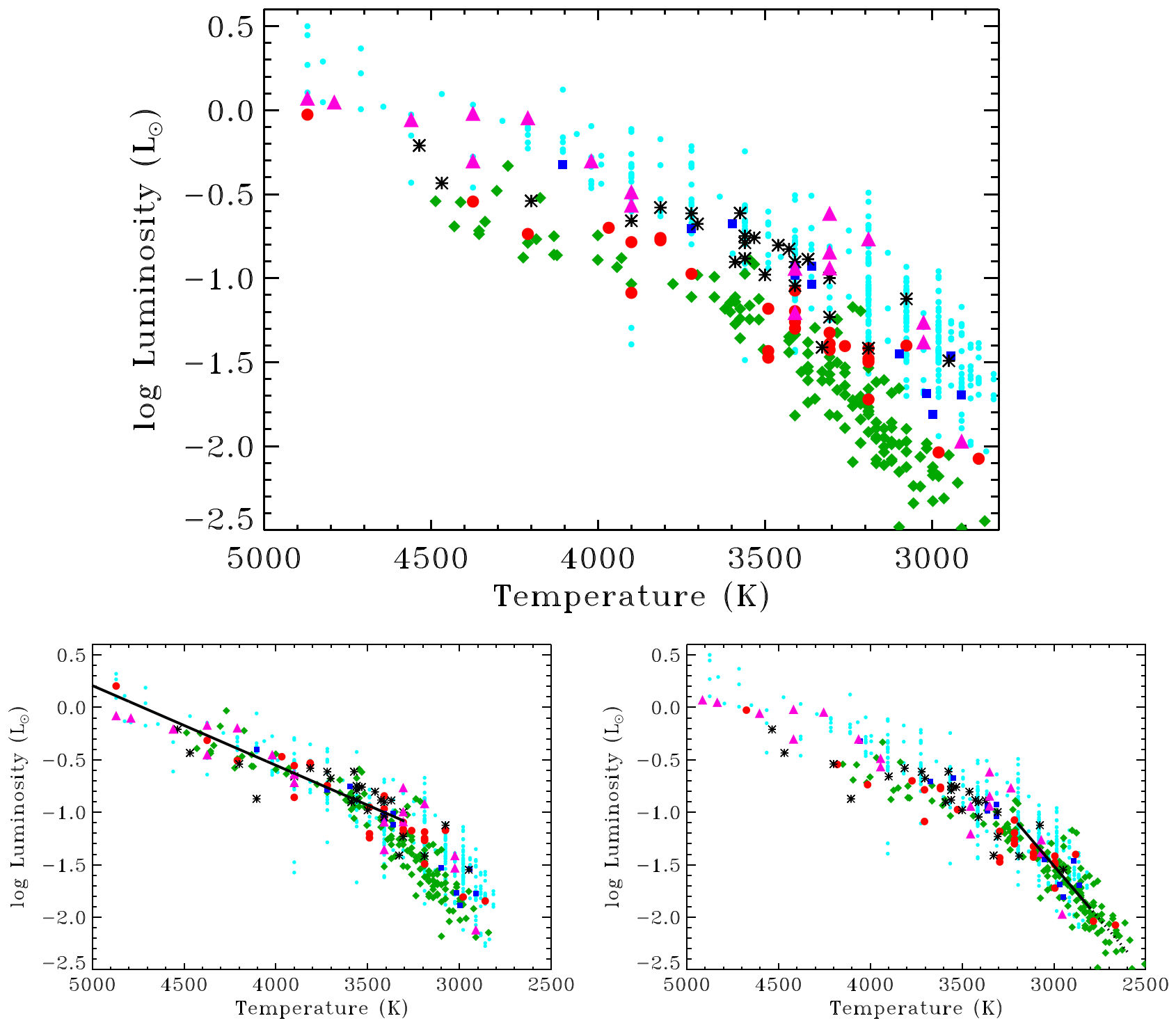

Figure 6. HR diagrams for nearby young associations. The top diagram shows the luminosity and temperature for members of the benchmark five associations (green diamonds for the Tuc Hor Moving Group, red circles for the $\beta$ Pic Moving Group, black asterisks for the TW Hya Association, blue squares for the $\eta$ Cha Association, purple triangles for the $\epsilon$ Cha Association, and small cyan circles for the Upper Sco OB Association). The bottom panels show the same figure, with the stars offset in luminosity (left) to calculate a best fit to the $3200-5000 \mathrm{~K}$ stars (black solid line) and offset in temperature (right) to calculate a best fit to the $2800-3200 \mathrm{~K}$ brown dwarfs (black solid line, extends to $<2800 \mathrm{~K}$ because some temperatures are shifted into this range).

The total combined fit to our benchmark clusters yields a slope of

$$
\log L / L_{\odot} \propto(2.05 \pm 0.15) T / 10^{3} \mathrm{~K}\left(T_{\text {infl }}-5000 \mathrm{~K}\right),
$$

where the temperature range goes from 2800 and $3200 \mathrm{~K}$ for the younger clusters and $2800-3400 \mathrm{~K}$ for the older clusters (as also described above with $\left.T_{\text {infl }}\right)$. The error in the slope is calculated as in Section 3.1 The fits are dominated by members of the Tuc Hor and $\beta$ Pic Moving Groups because those regions have more identified brown dwarf members. Fits are calculated for the combined locus of young associations but are not calculated for each individually because each association has only a few data points. Table 3 lists slopes for the individual regions and the combined HR diagram.

The coolest known objects in these regions $(<2800 \mathrm{~K})$ are fainter than expected from these linear fits. They are excluded in this analysis, but their inclusion would significantly steepen the slopes of these lines. This discrepancy suggests that these fits may not be robust to the selection of the low temperature cutoff for this fit.

The measured uncertainty in the linear fit of $0.15 \log L / L$ 。 per $10^{3} \mathrm{~K}$ leads to a 0.06 dex uncertainty in relative luminosity between a 3200 and $2800 \mathrm{~K}$ brown dwarf. The difference in slopes between the older and younger associations likely leads to 0.09 dex uncertainties over the same regions. This difference is likely real evolution in the brown dwarf loci and not related to uncertainty in the fits. In Section 3.4, comparisons to the Upper Sco OB Association demonstrate that the slope becomes shallower with age. The results from linear fits in producing a luminosity for a $3000 \mathrm{~K}$ brown dwarf may still be robust as long as the known membership is well distributed between 2800 and $3200 \mathrm{~K}$. However, this relationship should only be used with caution. 


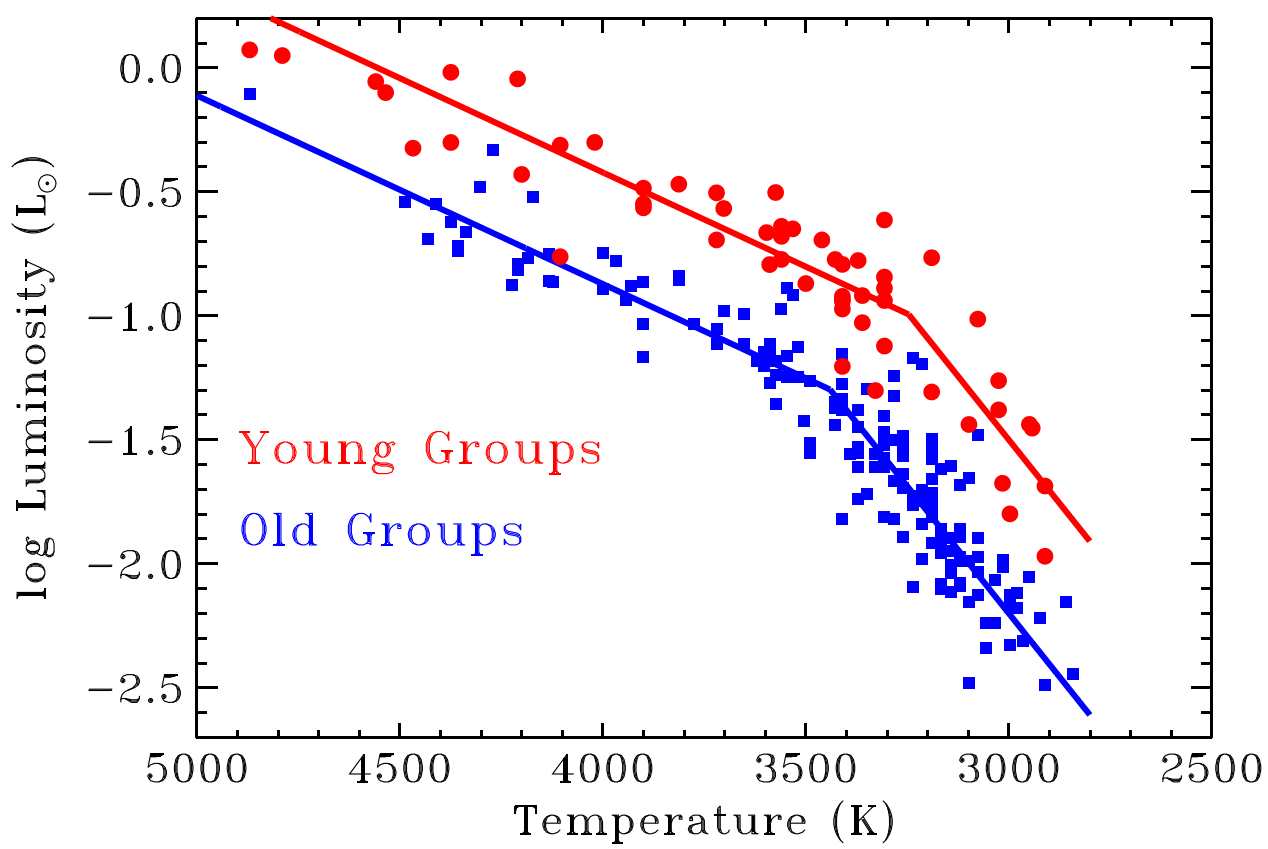

Figure 7. HR diagram for young (red circles) and old clusters (blue squares), with members of the $\beta$ Pic Moving Group shifted to overlap with the Tuc Hor stellar locus, while $\eta$ Cha and TW Hya Association members are shifted to overlap with the $\epsilon$ Cha Association stellar locus. The blue and red lines show the best fits to the combined low mass star and brown dwarf locus, also shifted to match the young and old groups.

The fit to the brown dwarf locus is not recalculated for Pecaut \& Mamajek (2013) parameters because their bolometric corrections are not calculated for spectral types later than M5.

\subsection{Combined Isochrones between 2800 and $5000 \mathrm{~K}$}

Subsections 3.1 and 3.2 describe empirical fits to HR diagrams, as illustrated in Figure 6. The regions warmer and cooler than the inflection point in the temperature-luminosity locus were fit separately, with one slope but different $y$-intercepts for each association. The shifts obtained from the $y$-intercepts are visualized as luminosity shifts, while the fits to the cooler stars are visualized as temperature shifts.

The location of the inflection point, which motivates the separate fits to the two temperature ranges, depends on the cluster age. In this subsection, we compare the independent results from the fits to low mass star and brown dwarf isochrones to describe how the empirical lines combine to describe the low mass locus of young clusters. For illustrative purposes only, the combined isochrones are plotted against the young and old associations in Figure 7.

Figure 8 shows the luminosity of $3000 \mathrm{~K}$ brown dwarfs versus $4200 \mathrm{~K}$ stars from our independent fits to the young clusters with a best fit line of

$$
\log \left(L_{4200} / L_{\odot}\right)=-1.02+1.54 \log \left(L_{3000} / L_{\odot}\right) .
$$

Combining the line of Equation (3) with the two slopes reported in Equations (1) and (2) fully describes the evolution of the empirical locus of single low mass stars and brown dwarfs in clusters. The isochrone for low mass stars falls in luminosity more quickly than that for brown dwarfs. The inflection point where the temperature-luminosity slope changes is determined by the intersection of the stellar and brown dwarf locus described by this line.

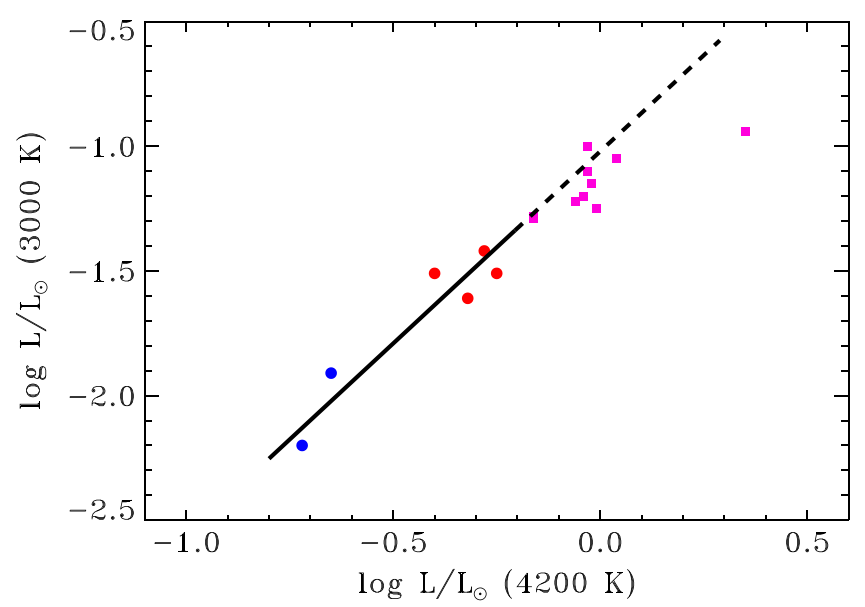

Figure 8. Relationship between the luminosity for 4200 and $3000 \mathrm{~K}$ stars based on the lines fit to the individual clusters. The blue and red circles are the old and young associations (including Upper Sco), respectively, and are best fit with the solid black line. The purple squares are preliminary results from younger clusters, most of which are fit by an extrapolation of the best fit line to higher luminosities.

As a further test of our method, Figure 8 also shows preliminary results of its application to younger clusters with proportionally higher luminosities. The data are obtained for IC 348, Chameleon I, Lupus I, Taurus, $\sigma$ Ori, $\lambda$ Ori, and the ONC (Luhman et al. 2003, 2010; Luhman 2007; Rebull et al. 2010; Bayo et al. 2011; Rigliaco et al. 2011; da Rio et al. 2012). Although the errors in the placement of individual stars on the HR diagram are larger for younger stars with more influence from disks and heavier extinction, their mean luminosities are encouragement that our method could be applied to younger regions in order to constrain the luminosity evolution for comparison to theoretical models. 

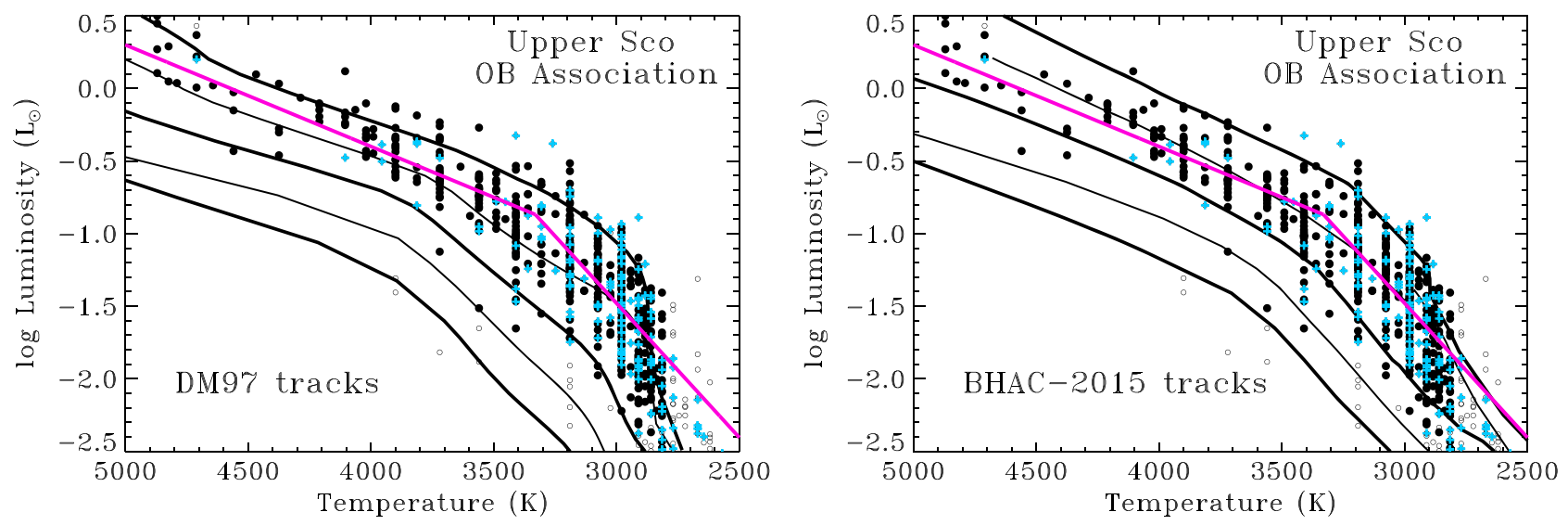

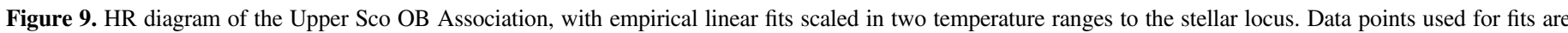

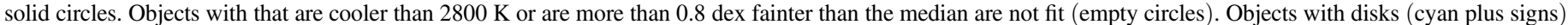

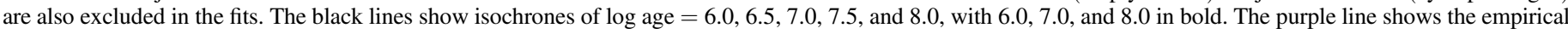
isochrone for low mass stars and brown dwarfs, scaled to the Upper Sco OB Association data.

\subsection{Application to the Upper Sco OB Association}

Figure 9 shows how the fits derived above are applied to the HR diagram of the Upper Sco OB Association. Lines with slopes that best fit the loci of low mass stars $(3200-5000 \mathrm{~K})$ and brown dwarf/very low mass stars $(2800-3200 \mathrm{~K})$ are scaled to the measured isochrone.

The initial sample includes stars with $K$-W2 color consistent with diskless stars and also stars without WISE detections. An initial scaling to the $3200-5000 \mathrm{~K}$ region (186 stars) has $\log L_{4200} / L_{\odot}=-0.28$ with a standard deviation of 0.26 dex, much larger than that obtained for the other clusters. Several stars are severe outliers, defined here as 0.5 dex from the $\log L$ calculated in the initial fit. Our adopted fit of 176 stars excludes these severe outliers. We calculate $\log L_{4200} / L_{\odot}=-0.23$ with a luminosity scatter of $0.18 \mathrm{dex}$. Fitting stars from $3400-5000 \mathrm{~K}$ yields the same $\log L_{4200}$. This luminosity spread of 0.18 dex is slightly larger than that calculated for the other associations, with extra scatter that may be caused by a real age spread in the cluster or, as suggested by Slesnick et al. (2008), $\mathrm{a} \sim 30 \mathrm{pc}$ depth of the region.

The brown dwarf locus of Upper Sco at 2800-3200 K has a much steeper slope than the linear fit to the 5-40 Myr old sample, which points to problems in our simplifying assumption that the slope of the 2800-3200 K object locus does not evolve on these timescales. The fit to the slope of combined loci of the five benchmark clusters is dominated by Tuc-Hor members. However, the slopes from the younger associations are steeper than for the older associations. These differences suggest that the empirical brown dwarf locus is better described by a swinging gate rather than a single slope. This same swinging gate behavior is also suggested by the theoretical isochrones covering this low temperature regime (Figure 9), where the predicted slopes appear to flatten with increasing age. In particular, the brown dwarf loci is remarkably well described by the DM97 isochrones and is also consistent with the BHAC-15 isochrones.

While a more in depth analysis is beyond the scope of the spirit of this paper, these problems with the brown dwarf isochrones lead us to focus subsequent analysis of the correspondence between stellar mean stellar luminosities and stellar ages on the 3200-5000 K stellar locus.

\subsection{Ordering Clusters by Luminosity}

In this section, we apply the analytical approximations of isochrones from Section 3.1 to 3.2 to the set of young clusters to order them in luminosity as a proxy for age. The fits to luminosities for $3200-5000 \mathrm{~K}$ stars (Section 3.1) are likely more accurate than for the $2800-3200 \mathrm{~K}$ brown dwarfs (Section 3.2) because an analysis of the brown dwarf locus relies heavily on the two older moving groups and may not accurately describe the brown dwarf locus of younger clusters.

Table 4 shows the ordering of clusters by luminosity for the $3200-5000 \mathrm{~K}$ fits. The five benchmark associations are ordered in luminosity as expected from previous studies (e.g., see discussions in Mamajek 2009; Hillenbrand 2009; Kraus et al. 2011 and Fang et al. 2013). The Upper Sco, $\epsilon$ Cha, and $\eta$ Cha Associations are the youngest, followed by the TW Hya Association, the $\beta$ Pic Moving Group, and the Tuc-Hor Moving Group. Use of Pecaut \& Mamajek (2013) temperatures and bolometric corrections would increase the association $\log L_{4200}$ by $0.02-0.03$ dex.

The formal uncertainties in the luminosity (age) ordering are calculated from a combination of (1) the standard deviation divided by the square root of the number of stars in the sample, and (2) systematic uncertainty in distances. Methodological differences in luminosity calculations and in the census of multiplicity also can affect the luminosity ordering of clusters. For the specific benchmark clusters analyzed here, the age ordering is secure, with the possible exception of the $\eta$ Cha Association. To swap the TWA and $\eta$ Cha Associations would require a systematic change in $L_{4200}$ by $0.08 \mathrm{dex}(20 \%)$. For young clusters, the primary culprits to explain such a luminosity change of $0.1 \mathrm{dex}$ would be systematic errors in the distance (12\% change), extinction (change of $A_{V}=0.9$ mag), and spectral type (change of $140 \mathrm{~K}$ ). Weinberger et al. (2013) argue that TWA 9 A and 9 B should be excluded from the TWA census because they are much fainter than expected, which would increase the cluster luminosity by 0.04 dex. These comparisons are also affected by differences in binary accounting and by accretion, if samples include disk sources.

\section{COMPARING EMPIRICAL AND MODEL ISOCHRONES}

The relationship between stellar luminosity and effective temperature has been measured for six young associations. 


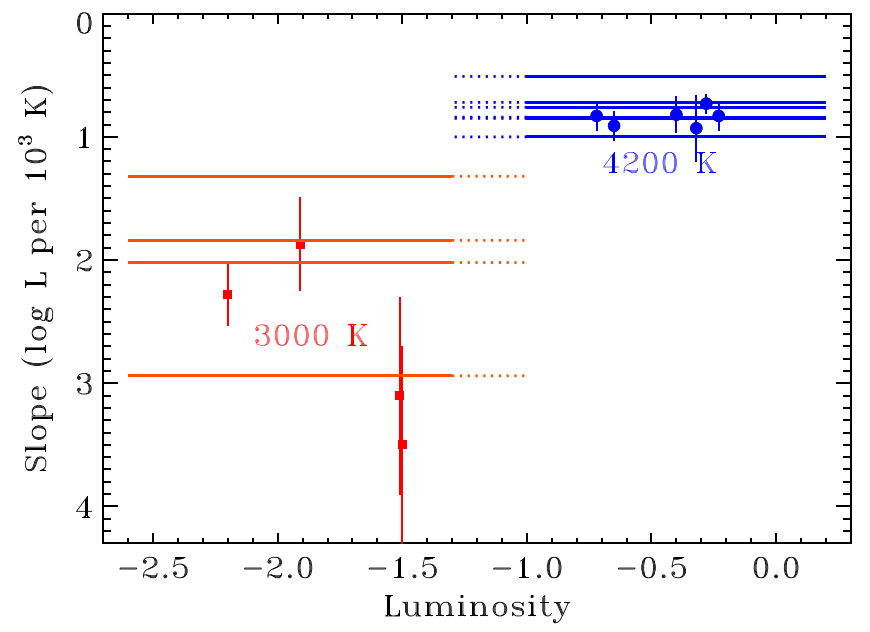

Figure 10. Measured slopes of luminosity vs. temperature for each association (blue circles for 3200-5000 K stars with luminosities assessed at $4200 \mathrm{~K}$; red circles for brown dwarfs with luminosities assessed at $3000 \mathrm{~K}$ ) compared with slopes predicted from Feiden, BHAC-15, Pisa, DM97, PARSEC, and Siess tracks evaluated at $20 \mathrm{Myr}$ (see Tables 3 and 4 for values). The solid lines show the luminosity interval over which the slopes apply for both the young and old associations in this paper. The dotted lines show the intermediate regime where the inflection point occurs. The brown dwarf points include one slope and luminosity calculated for the combined young associations.

Because these stars are contracting pre-main-sequence stars, the radius and therefore luminosity decreases with time. The luminosity of stars at a fixed temperature is therefore used in Section 3.5 as a proxy for age. The conversion from luminosity to age depends on the adopted choice among the many evolutionary models, as shown in Figure 3, and on the stellar temperature. At a fixed temperature, the age estimates differ between the many evolutionary models. Mismatches between empirical and model isochronal slopes lead to different age estimates along an empirical isochrone when a given model family is applied to stars with different temperatures.

Figures 10 and 11 and Table 3 compare the empirical isochrones to the pre-main-sequence models discussed in this paper. The model isochrones are curved but can be well approximated with a two temperature fit within the $2800-5000 \mathrm{~K}$ region. The slopes presented in Table 3 are intended as a guide, with a measured slope that is sensitive to the temperature region defined in the fit. For low mass stars (3200-5000 K), the luminosity slopes in the Dartmouth, Pisa, BCAH98-1.9, BHAC-2015, and Feiden pre-main-sequence tracks are consistent with the observations. These models produce similar ages for stars at 3800 and $4200 \mathrm{~K}$. On the other hand, the isochronal slopes predicted from the DM97, Siess, BCAH98, and PARSEC models differ from empirical slopes. This discrepancy leads to differences in expected luminosities versus spectral type. The DM97, Siess, and BCAH98 models all have a steeper slope than is observed, which leads to a temperature (mass) dependence in age estimates. As an example, if we normalize our empirical isochrone and the BCAH98 isochrone to a young $4200 \mathrm{~K}$ star, a co-eval star of $3400 \mathrm{~K}$ would appear 0.3 dex more luminous ( 0.4 dex younger in age) than expected from the BCAH98 isochrone. The PARSEC isochrones conversely have a shallower slope than is observed, which leads to $3400 \mathrm{~K}$ stars appearing older than $4200 \mathrm{~K}$ stars.
The new BHAC-2015 and Feiden models have isochrones that are similar to the empirical isochrones. The inflection point in both BHAC-2015 and Feiden occurs at temperatures $\sim 50-150 \mathrm{~K}$ hotter than in the empirical isochrones, which leads to mismatches between empirical and model isochrones for very low mass stars and brown dwarfs. These two models predict isochrones that are close enough to empirical isochrones that the differences could be explained if effective temperatures below $3500 \mathrm{~K}$ are systematically underestimated by $50-100 \mathrm{~K}$.

Given these mismatches between model and observed isochrones, even for the latest models for cool temperatures, comparisons between clusters should be made at a constant temperature. Age estimates for each model are evaluated at 3400,3800 , and $4200 \mathrm{~K}$ and are plotted in Figure 12 for three of the benchmark associations and for the Upper Sco OB Association. Because of the discrepancies in slope described above, for most models the $3400 \mathrm{~K}$ stars are $0.2-0.5 \mathrm{dex}$ younger than 3800 and $4200 \mathrm{~K}$ stars. The PARSEC models have the reverse dependence, with the $3400 \mathrm{~K}$ stars older than the $4200 \mathrm{~K}$ stars.

The brown dwarf ages are not plotted. For the now-outdated BCAH98 models, ages of $3000 \mathrm{~K}$ stars are another $0.3 \mathrm{dex}$ younger than the $3400 \mathrm{~K}$ stars. On the other hand, for PARSEC models ages of $3000 \mathrm{~K}$ stars are typically $0.1-0.3$ dex older than $3400 \mathrm{~K}$ stars. However, the differences between the brown dwarf loci of Upper Sco OB Association and the Tuc Hor Moving Group demonstrates that the shape and slope of the brown dwarf isochrone evolves with time. Fitting a single slope to the brown dwarf isochrones without respect to age is an overly simplistic approach to characterizing the location of brown dwarfs in the HR diagram. The DM97 models reproduce the evolution in the brown dwarf isochones especially well, however the absolute ages are anomalously young.

\section{DISCUSSION}

Converting the luminosity proxy into a consistent and accurate tool for age measurements requires solving two problems: (1) establishing whether the ages inferred from luminosities are consistent across a temperature range, and (2) comparing the model-dependent age estimates with independent age estimates to establish an absolute and correct age scale.

The empirical isochrone fits presented here lead to a selfconsistent luminosity ordering for nearby young associations. Absolute ages are then estimated from comparisons to isochrones for model tracks, but these comparisons lead to a wide range of age estimates for different model tracks and within the same models for stars of different temperatures. If uncorrected, these discrepancies lead to significant spectral type (mass) dependences in ages of young stars.

The temperature dependency of the inferred ages arises from the mismatch between empirical and theoretical slopes in $\log L$ versus temperature. The source for this mismatch may be the evolutionary models, although spectral type/temperature mismatches between different methodologies and uncertainties in the spectral type/temperature conversion also likely contribute to these discrepancies. The BHAC-2015 models improved the prescription for convection, while the Feiden models improve upon the thermal structure in the atmosphere. Both the BHAC2015 and Feiden models significantly improve the match between predicted and observed stellar loci. Alternately, the PARSEC models apply an ad hoc change to the T $-\tau$ relationship 

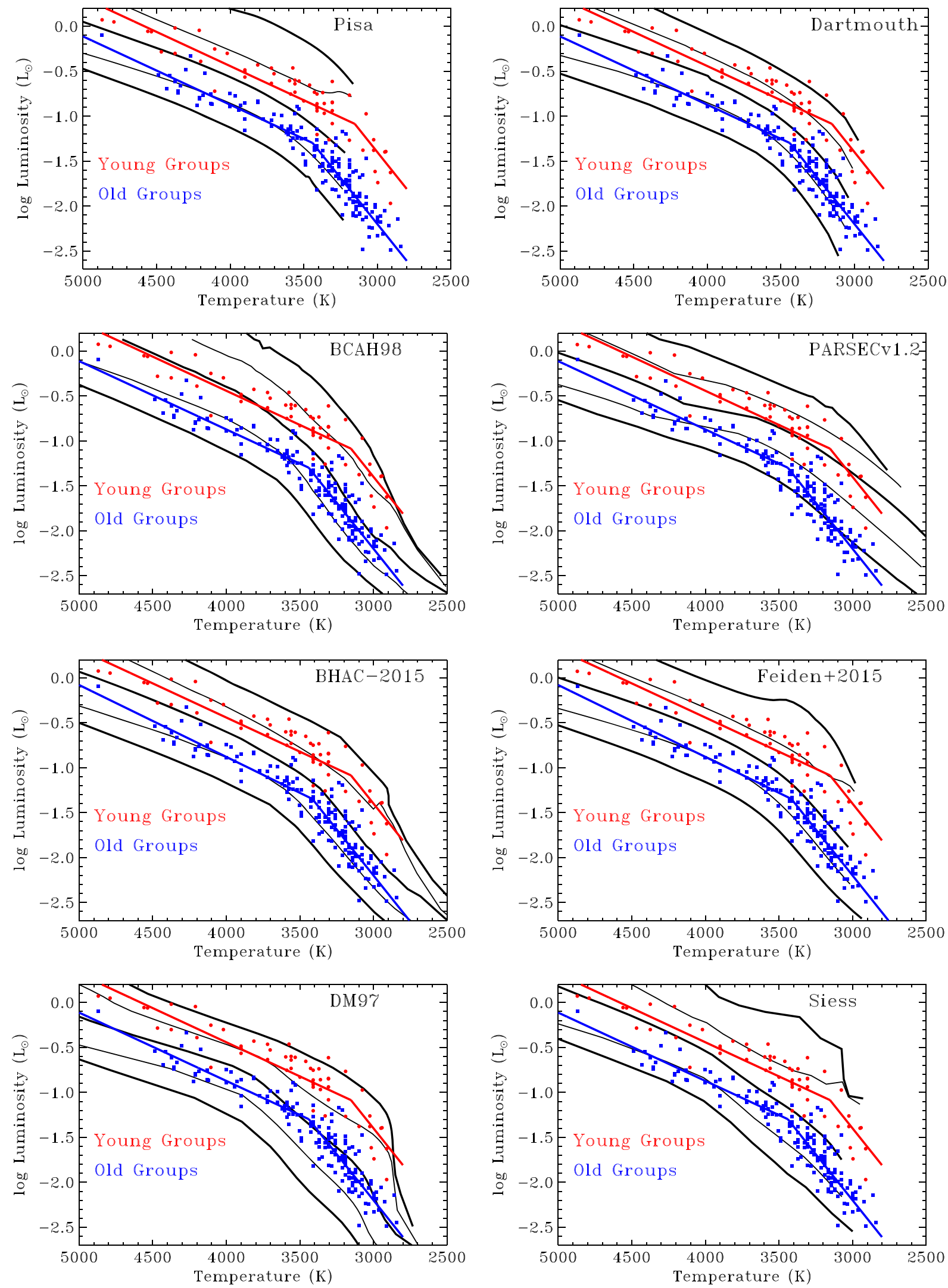

Figure 11. Empirical HR diagrams, along with two-temperature regime linear fits, compared to isochrones from pre-main-sequence evolution models. The points for $\eta$ Cha and $\epsilon$ Cha Association members are shifted to be consistent with the isochrone for TW Hya, while the $\beta$ Pic Moving Group members are shifted to be consistent with Tuc-Hor Moving Group members. As in Figure 8, the black lines show isochrones of log age =6.0, 6.5, 7.0, 7.5, and 8.0, with 6.0, 7.0, and 8.0 in bold. 

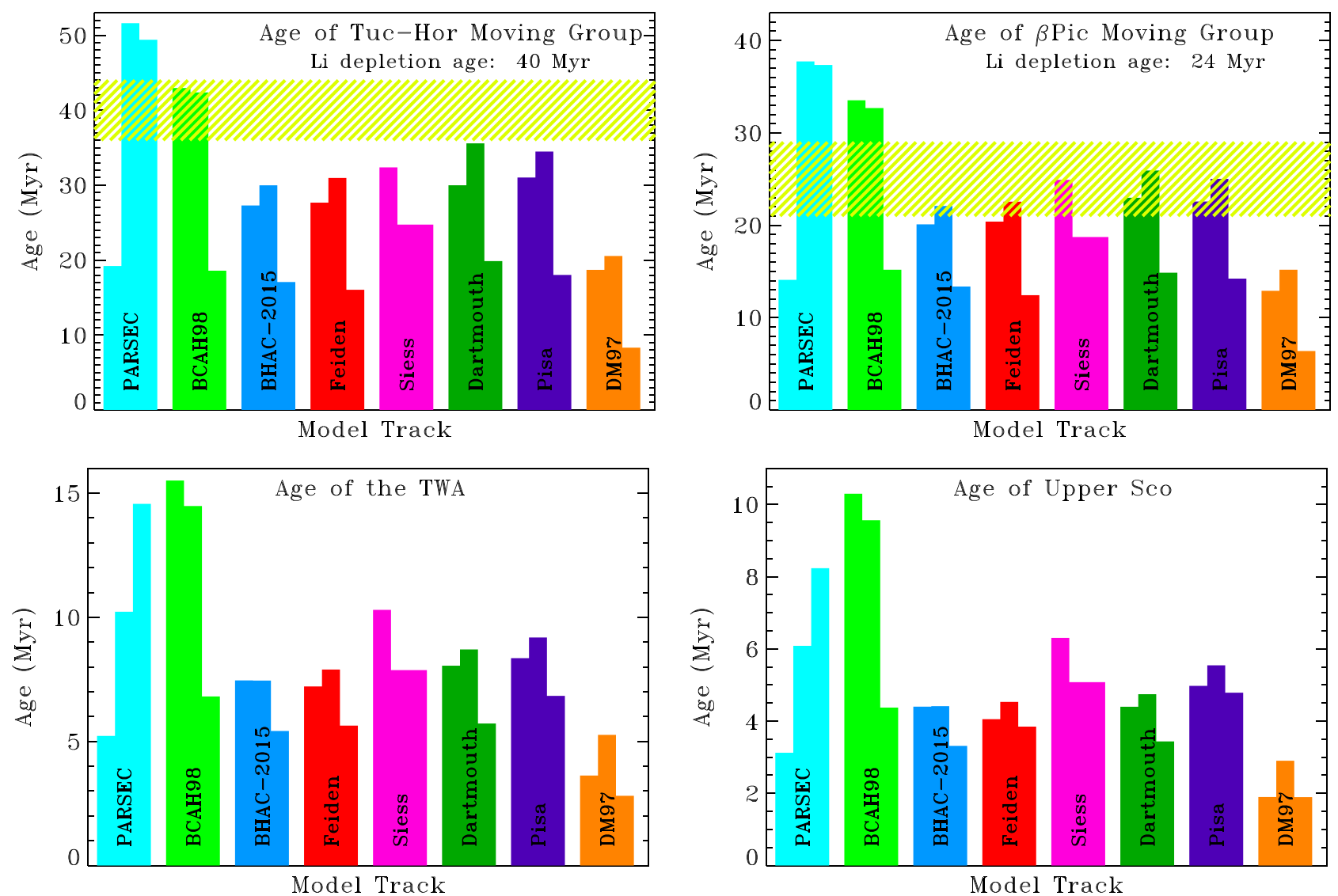

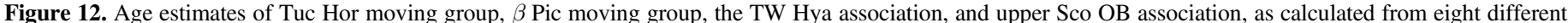

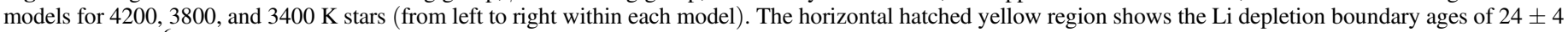
and $40 \pm 4 \mathrm{Myr}^{6}$ for the $\beta$ Pic and Tuc-Hor Moving Groups (Binks \& Jeffries 2014; Kraus et al. 2014; Malo et al. 2014).

to fit the color constraints of old clusters, and similar tweaks may be able to reproduce loci of low mass young stars.

Ultimately, despite recent advances, some model improvements are still necessary so that the evolution of radius and temperature better reflects the observational constraints of young stellar associations. Missing physics in the evolutionary models, including magnetic fields and spots, may lead to mass dependent discrepancies or absolute errors in age dating young clusters. Age comparisons between clusters are self-consistent only when compared over specific temperature ranges where the model and empirical isochrones match. The BHAC-2015 and Feiden models currently offer the best set of pre-main sequence tracks for relative age estimates, although mismatches at $<3500 \mathrm{~K}$ still lead to temperature-dependent ages. The empirical isochrones derived in this paper offer a method to place stars of different temperatures on the same age scale, albeit with some uncertainty introduced by the possible temporal evolution of the isochronal slopes.

Table 5 lists sets of ages for each cluster based on a consistent application of a single model family at a single stellar temperature. The ages are selected from models that produce young, intermediate, and old ages relative to the average model. Uncertainties in these ages are calculated from the statistical uncertainties in the fits, as listed in Table $4,{ }^{9}$ and an estimate for the systematic uncertainty in the median distance of the $\eta$ Cha, $\epsilon$ Cha, and Upper Sco OB Associations.

\footnotetext{
9 The luminosity errors in dex are asymmetric in linear space. The listed errors are the median error in Myr.
}

The reasonableness of these isochronal ages are testable by comparison to Li depletion boundary ages. ${ }^{10}$ The Tuc-Hor Moving Group has a Li depletion boundary age of $41 \pm 2 \mathrm{Myr}$ from the BCAH98 evolutionary models and $38 \pm 2$ Myr from the DM97 models (Kraus et al. 2014), so we adopt $40 \pm$ 4 Myr. The $\beta$ Pic Moving Group has Li depletion boundary ages of $21 \pm 4$ Myr from (Binks \& Jeffries 2014) and $26 \pm$ 3 Myr from the Feiden models (Malo et al. 2014). We adopt an Li depletion boundary age of $24 \pm 4 \mathrm{Myr}$ for the $\beta$ Pic Moving Group.

Figure 12 and Table 5 compares the Li depletion boundary ages to the isochronal ages estimated here from the different model tracks. No single model of low mass stars reproduces the Li depletion boundary age for both the Tuc-Hor and $\beta$ Pic Moving Groups. Most models underpredict the age of the TucHor Moving Group by $\sim 10 \mathrm{Myr}$ (or $0.1 \mathrm{dex}$ ). On the other hand, most models reproduce the age of the $\beta$ Pic Moving Group. The DM97 models yield especially young ages, likely because the isochrones of low mass stars have temperatures that are initially too high (see description in Section 2.5). Both the Feiden and BHAC-2015 models have similar isochrones that yield remarkably similar ages.

\footnotetext{
10 The Li depletion boundary ages may be systematically overestimated by a few Myr if the radius dispersion within a cluster decreases the timescales for Li depletion (Somers \& Pinsonneault 2014). On the other hand, inclusion of magnetic spots may lead to $30 \%$ and $20 \%$ underestimates of the ages of the $\beta$ Pic and Tuc-Hor Moving Groups, respectively (Jackson \& Jeffries 2014).
} 
Table 5

Consistent Sets of Ages (in Myr) of Young Associations ${ }^{\mathrm{a}}$

\begin{tabular}{|c|c|c|c|c|c|c|c|c|}
\hline Stellar & & & DM97 & Pisa & BCAH98 & Feiden & BHAC-2015 & Li Depl. $^{\text {b }}$ \\
\hline Association & $d(\mathrm{pc})$ & $\log L_{4200} / L_{\odot}$ & $3400 \mathrm{~K}$ & $4200 \mathrm{~K}$ & $4200 \mathrm{~K}$ & $3800 \mathrm{~K}$ & $3800 \mathrm{~K}$ & Age \\
\hline Upper Sco & $145 \pm 10^{\mathrm{c}}$ & $-0.25 \pm 0.06$ & $1.7 \pm 0.2$ & $5.0 \pm 1.1$ & $10.3 \pm 1.9$ & $4.2 \pm 1.0$ & $4.1 \pm 1.0$ & $\cdots$ \\
\hline$\epsilon$ Cha & $111 \pm 6^{\mathrm{c}}$ & $-0.28 \pm 0.06$ & $1.9 \pm 0.3$ & $5.6 \pm 1.4$ & $11.3 \pm 2.1$ & $4.7 \pm 1.2$ & $4.7 \pm 1.2$ & $\cdots$ \\
\hline$\eta$ Cha & $94 \pm 5^{c}$ & $-0.32 \pm 0.05$ & $2.1 \pm 0.3$ & $6.6 \pm 1.3$ & $12.7 \pm 2.1$ & $5.5 \pm 1.3$ & $5.5 \pm 1.3$ & $\cdots$ \\
\hline TWA & $(57)$ & $-0.40 \pm 0.04$ & $2.7 \pm 0.4$ & $9.0 \pm 1.5$ & $16.5 \pm 2.1$ & $7.9 \pm 1.4$ & $8.1 \pm 1.4$ & $\cdots$ \\
\hline BPMG & $(30)$ & $-0.65 \pm 0.04$ & $6.1 \pm 0.7$ & $24 \pm 4$ & $36 \pm 5$ & $23 \pm 4$ & $22 \pm 4$ & $24 \pm 4$ \\
\hline Tuc Hor & $(51)$ & $-0.72 \pm 0.02$ & $7.7 \pm 0.5$ & $32 \pm 3$ & $44 \pm 3$ & $30 \pm 3$ & $29 \pm 3$ & $40 \pm 4$ \\
\hline
\end{tabular}

Note.

${ }^{a}$ For models that produce a younger, intermediate, and old age, plus the recent BHAC-2015 and Feiden models.

${ }^{\mathrm{b}}$ Li depletion boundary ages, see text for details.

${ }^{\mathrm{c}}$ Estimated uncertainty in median cluster distances.

When applied consistently, the Li depletion boundary ages for the $\beta$ Pic Moving Group implies a 4 Myr old age for low mass stars in the Upper Sco OB Association, younger than the $11 \mathrm{Myr}$ age measured from intermediate and high mass stars by Pecaut et al. (2012). The $11 \mathrm{Myr}$ age of Upper Sco would require ages of $\sim 40$ and $55 \mathrm{Myr}$ for the $\beta$ Pic and Tuc-Hor Moving Groups. Either the intermediate mass or low mass age (based on Li depletion boundary ages of older associations) of the Upper Sco OB Association is incorrect, or the identification and evaluation of the low mass and/or higher mass stars is biased in some way.

Errors in ages may be caused by either errors in the birthline or in the contraction rates. A bulk subtraction of $5 \mathrm{Myr}$ to intermediate mass isochrones would shift the intermediate mass isochrones sufficiently for Upper Sco and would not cause any significant discrepancy with the Li depletion age of the $\beta$ Pic Moving Group. The age estimate for the $\beta$ Pic Moving Group from late F and G stars (Mamajek \& Bell 2014) would also not change significantly because these stars are preferentially lower mass than the intermediate/high mass stars that led to the old age estimate for the Upper Sco OB Association. An addition of $5 \mathrm{Myr}$ to low mass isochrones would also lead to consistent ages, but would encounter severe problems by forcing the youngest known clusters to have ages of $5 \mathrm{Myr}$. Alternately, the contraction rates for either low mass or intermediate mass stars may be in error. For low mass stars, the inclusion of magnetic fields and spots in evolutionary models will affect contraction rates. For intermediate and high mass stars, the inclusion of rotation leads to older ages for intermediate and high-mass stars.

Observational errors relative to the benchmark associations may also lead to an underestimate of the age. A bulk increase in temperatures by $250 \mathrm{~K}$ would lead to an age of $10 \mathrm{Myr}$, but such a large systematic difference in spectral typing is unlikely. Alternately, the luminosities could be overestimated by $0.2 \mathrm{dex}$ if the distances are biased and overestimated by $25 \%$. Assuming $A_{V}=0 \mathrm{mag}$, which disagrees with the colors of Upper Sco stars, would lead to only a 0.07 dex increase in the luminosities.

\section{CONCLUSIONS}

In this paper, we calculate empirical isochrones in HR diagrams for low mass stars and brown dwarfs in young (5-40 Myr) nearby associations. The sample consists of stars that have accurate distances, low extinction, and are not affected by the presence of disks or accretion. The simple formulation of the empirical isochrone assumes that two slopes, one applied to stars with temperatures 3200/3400-5000 K and one for brown dwarfs between 2800 and 3200/3400 K, applies to the stellar locus of all young associations. This choice leads to reasonable fits to the five young benchmark clusters and to the stellar and brown dwarf locus of the Upper Sco OB Association.

A successful theory of pre-main-sequence evolution should match these empirical fits. The Dartmouth, Pisa, and BCAH981.9 pre-main-sequence isochrones between 3200 and 5500 are consistent with the measured slopes. The slopes from DM97, BCAH98, and Siess isochrones are steeper than observed, so that very low mass stars will be assessed as younger than solar mass stars. The PARSEC isochrones have the opposite behavior, so that solar mass stars are assessed as younger than very low mass stars. The PARSEC and BCAH98 models typically lead to the oldest ages, while the ages obtained from the DM97 models are anomalously young. Advances in prescriptions for convection and the thermal structure of the atmosphere in the BHAC-2015 and Feiden models, respectively, have improved the match to empirical stellar loci at $>3500 \mathrm{~K}$ and match the decrease in luminosity at $<3500 \mathrm{~K}$, though with a temperature offset relative to established spectral type-temperature conversions.

Applying the empirical isochrone to associations provides a statistically robust age ordering, which is independent of spectral type. Our age ordering is based on the $3200-5000 \mathrm{~K}$ region. The assumption that the $2800-3200 \mathrm{~K}$ isochrones have the same slope for both the young and old associations is likely overly simplistic. Steeper slopes at younger ages, especially for the Upper Sco OB Association, instead indicates some evolution in the brown dwarf locus. Because the relationship between luminosity and temperature for brown dwarfs is steep, any use of brown dwarfs in age estimates requires accurate temperatures and should be used with caution.

Fitting these isochrones to the six associations discussed in this paper leads to a luminosity/age ordering mostly as expected from previous results, with the Upper Sco $\left(L_{4200} / L_{\odot}=-0.23\right), \eta$ Cha $(-0.28)$, and $\epsilon$ Cha $(-0.32)$, Associations roughly co-eval, followed by the TW Hya Association $(-0.40)$ and the $\beta$ Pic $(-0.65)$ and Tuc-Hor $(-0.72)$ Moving Groups. For any given association, age estimates of different models evaluated at different temperatures span 0.8 dex. As a consequence, comparisons of ages between different associations should consistently apply a single luminosity-age scaling. The BHAC-2015 and Feiden pre-main sequence models yield very similar ages for low mass stars. Ages can be adopted to be consistent with the $\mathrm{Li}$ depletion boundary age of the $\beta$ Pic Moving Group, but these 

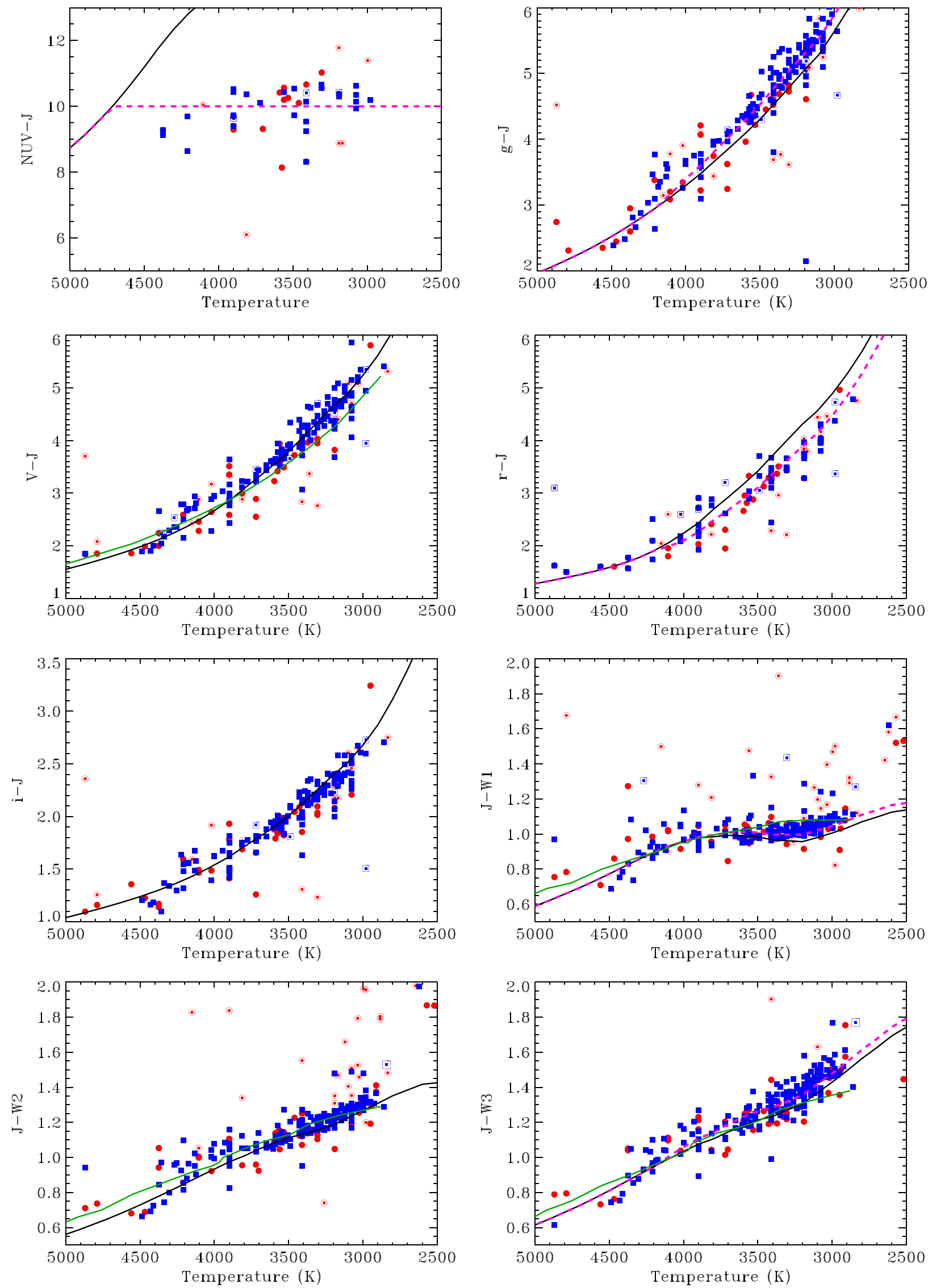

Figure 13. Color-temperature plots for members of young (red circles) and old (blue squares) nearby associations. All colors include $J$ to evaluate bolometric corrections from the $J$ band. Synthetic colors from BT-Settl models are plotted as the black line, while colors for young (5-30 Myr) stars from Pecaut \& Mamajek (2013) are plotted in green. In cases where the observed colors deviate from the model colors, the colors adopted for the bolometric correction calculations are shown as a dashed purple line. Objects with disks, identified from a $K-\mathrm{W} 2$ or $K-\mathrm{W} 3$ excess (Figure 2), are shown here as the small filled circles or squares surrounded by an outer circle or square. 
models imply a $4 \mathrm{Myr}$ age for the Upper Sco OB assocation for low mass stars, younger than the $11 \mathrm{Myr}$ age obtained from intermediate mass and high mass stars stars.

We thank the referee, Cameron Bell, for a careful read of the manuscript, which led to substantial improvements in the robustness and clarity of results and in the self-consistency within the paper. G.J.H. thanks Adam Kraus for discussions on preparing the paper and Jessy Jose for valuable comments on the paper. G.J.H. is also grateful for the role of the 2014 Oort Workshop on Episodic Accretion, jointly organized by Ewine van Dishoeck and Neal Evans, in motivating this analysis, and discussions with Lee Hartmann and Isabelle Baraffe at the workshop on early ideas for this paper. We appreciate Gregory Feiden sharing the new Dartmouth tracks prior to their publication and for discussion of those tracks and magnetic fields. G.J.H. is supported by a Youth Qianren grant and general grant \# 11473005 awarded by the National Science Foundation of China. This research has made use of the VizieR catalogue access tool, CDS, Strasbourg, France.

\section{APPENDIX \\ TARGET PHOTOMETRY AND BOLOMETRIC CORRECTIONS}

In Sections 2.2-2.3, we describe the collection and use of photometry to identify disk sources, to tweak bolometric corrections calculated from models, and to measure extinctions for members of the Upper Sco OB association. This Appendix describes how the photometry is implemented in this paper.

Filter curves and zero points are obtained from the Virtual Observatory Filter Profile Service. ${ }^{11}$ The BT-Settl models were convolved with the filter transmission profiles in photon space, which may lead to minor offsets relative to integrations in energy space (Cohen et al. 2003). The number of counts is then converted into a magnitude from the filter zero point function. Synthetic photometry is then calculated from the zero-point flux for the filter and the central wavelength of the filter. The listed central wavelengths are the average wavelength of the filter, calculated by integrating the transmission over the filter bandpass in wavelength space.

Figure 13 shows temperature-color plots that compare the observed photometry to synthetic photometry from the BT-Settl models and, when possible, to photometry of Pecaut \& Mamajek (2013). In cases where the observed and synthetic colors (always relative to $J$ ) are mismatched, the model fluxes at the corresponding wavelength are corrected to reproduce the observed colors. The $J$-band flux is always kept constant, so all corrections are relative to the $J$ band. The bolometric corrections are then calculated from the synthetic $J$-band photometry and the integrated emission in the full model spectrum.

Finally, the absolute bolometric magnitude $M_{\mathrm{bol}}$ is calculated from the distance-corrected $J$-band magnitude, $M_{J}$, and the bolometric correction, $\mathrm{BC}, M_{\mathrm{bol}}=M_{J}+B C$. The zero point

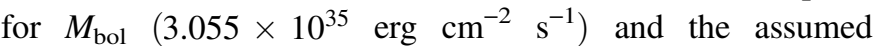
$L_{\odot}=3.83 \times 10^{33} \mathrm{erg} \mathrm{cm}^{-2} \mathrm{~s}^{-1}$ are obtained from the 1999 IAU Zero Point Scale, as discussed at the webpage of Eric Mamajek listing Basic Astronomical Data for the Sun. ${ }^{12}$

\footnotetext{
11 http://voservices.net/filter/

12 https://sites.google.com/site/mamajeksstarnotes/basic-astronomical-datafor-the-Sun
}

\section{REFERENCES}

Alcala, J. N., Natta, A., Manara, C. F., et al. 2014, A\&A, 561, 2

Allard, F., Homeier, D., \& Freytag, B. 2012, RSPTA, 370, 2765

Andrews, S. M., Rosenfeld, K. A., Kraus, A. L., \& Wilner, D. J. 2013, ApJ, 771,129

Asplund, M., Grevesse, N., Saucal, A. J., \& Scott, P. 2009, ARA\&A, 47, 481 Baraffe, I., Chabrier, G., Allard, F., \& Hauschlidt, P. H. 1998, A\&A, 337, 403

Baraffe, I., Chabrier, G., Barman, T. S., Allard, F., \& Hauschildt, P. H. 2003, A\&A, 402, 701

Baraffe, I., Chabrier, G., \& Gallardo, J. 2009, ApJL, 702, L27

Baraffe, I., Hormeier, D., Allard, F., \& Chabrier, G. 2015, A\&A, 577, 42

Bayo, A., Barrado, D., Stauffer, J., et al. 2011, A\&A, 536, 63

Bell, C. P. M., Naylor, T., Mayne, N. J., Jeffries, R. D., \& Littlefair, S. P. 2012, MNRAS, 424, 3178

Bell, C. P. M., Naylor, T., Mayne, N. J., Jeffries, R. D., \& Littlefair, S. P. 2013 , MNRAS, 434, 806

Bell, C. P. M., Rees, J. M., Naylor, T., et al. 2014, MNRAS, 445, 3496

Bianchi, L., Herald, J., Efremova, B., et al. 2011, Ap\&SS, 335, 161

Biller, B., Allers, K., Liu, M., Close, L. M., \& Dupuy, T. 2011, ApJ, 730, 39

Binks, A. S., \& Jeffries, R. D. 2014, MNRAS, 438, L11

Brandeker, A., Jayawardhana, R., Khavari, P., Haisch, K. E., \& Mardones, D. 2006, ApJ, 652, 1572

Bressan, A. G., Marigo, P., Girardi, L., et al. 2012, MNRAS, 427, 127

Caffau, E., Ludwig, H.-G., Steffen, M., Freytag, B., \& Bonifacio, P. 2011, SoPh, 268, 255

Chen, Y., Girardi, L., Bressan, A., et al. 2014, MNRAS, 444, 2525

Cohen, M., Wheaton, Wm. A., \& Megeath, S. T. 2003, AJ, 126, 1090

Covino, S., Lazzati, D., Ghisellini, G., et al. 1997, A\&A, 348, 187

Cutri, R. M., et al. 2012, yCat, 2311, 0

da Rio, N., Robberto, M., Hillenbrand, L. A., Henning, T., \& Stassun, K. G. 2012, ApJ, 748, 14

da Rio, N., Robberto, M., Soderblom, D. R., et al. 2010, ApJ, 722, 1092

D'Antona, F., \& Mazzitelli, I. 1997, MmSAI, 68, 807

D’Orazi, V., Biazzo, K., \& Randich, S. 2011, A\&A, 526, A103

de Zeeuw, P. T., Hoogerwerf, R., de Bruijne, J. H. J., Brown, A. G. G., \& Blaauw, A. 1999, AJ, 117, 354

Dotter, A., Chaboyer, B., Jevremovic, D., Kostov, V., Baron, E., \& Ferguson, J. W. 2008, ApJS, 178, 89

Ducourant, C., Teixeira, R., Galli, P. A. B., et al. 2014, A\&A, 563, 121

Fang, M., van Boekel, R., Bouwman, J., et al. 2013, A\&A, 549, 15

Gennaro, M., Prada Moroni, P. G., \& Pisa, E. 2012, MNRAS, 420, 986

Grevesse, N., \& Sauval, A. J. 1998, SSRv, 85, 161

Hartigan, P., \& Kenyon, S. J. 2003, ApJ, 583, 334

Hartigan, P., Morse, J. A., \& Raymond, J. 1994, ApJ, 436, 125

Hartmann, L., Cassen, P., \& Kenyon, S. J. 1997, ApJ, 475, 770

Hauschildt, P. H., Allard, F., \& Baron, E. 1999b, ApJ, 512, 377

Hauschildt, P. H., Allard, F., Ferguson, J., Baron, E., \& Alexander, D. R. 1999a, ApJ, 525, 871

Herczeg, G. J., \& Hillenbrand, L. A. 2014, ApJ, 786, 97

Hillenbrand, L. A. 1997, AJ, 113, 1733

Hillenbrand, L. A. 2009, in IAU Symp. 258, The Ages of Stars, ed. E. E. Mamajek, D. R. Soderblom \& R. F. G. Wyse (Cambridge: Cambridge Univ. Press), 81

Hillenbrand, L. A., Bauermeister, A., \& White, R. J. 2008, in ASP Conf. Ser. 384, 14th Cambridge Workshop on Cool Stars, Stellar Systems, and the Sun, ed. G. T. van Belle (San Francisco, CA: ASP), 200

Jackson, R. J., \& Jeffries, R. D. 2014, MNRAS, 445, 4306

Janson, M., Hormuth, F., Bergfors, C., et al. 2012, ApJ, 754, 44

Kraus, A. L., Ireland, M. J., Martinache, F., \& Hillenbrand, L. A. 2011, ApJ, 731,8

Kraus, A. L., Ireland, M. J., Martinache, F., \& Lloyd, J. P. 2008, ApJ, 679,762

Kraus, A. L., \& Hillenbrand, L. A. 2009, ApJ, 704, 531

Kraus, A. L., \& Hillenbrand, L. A. 2012, ApJ, 757, 141

Kraus, A. L., Shkolnik, E. L., Allers, K. N., et al. 2014, AJ, 147, 146

Lafreniere, D., Jayawardhana, R., van Kerkwijk, M. H., Brandeker, A., \& Janson, M. 2014, ApJL, 785, L47

Laskar, T., Soderblom, D. R., Valenti, J. A., \& Stauffer, J. R. 2009, ApJ, 698,660

Lopez Marti, B., Jiminez Esteban, F., Bayo, A., et al. 2013, A\&A, 551, 46 Luhman, K. L., Stauffer, J. R., Muench, A. A., et al. 2003, ApJ, 593, 1093

Luhman, K. L., \& Steeghs, D. 2004, ApJ, 609, 917

Luhman, K. 2007, ApJS, 173, L104

Luhman, K. L., Allen, P. R., Espaillat, C., Hartmann, L., \& Calvet, N. 2010, ApJS, 186, L111 
Luhman, K. L., \& Mamajek, E. E. 2012, ApJL, 758, 31

Malo, L., Artigau, E., Doyon, R., et al. 2014, ApJ, 788, 81

Malo, L., Doyon, R., Feiden, G. A., et al. 2014, ApJ, 792, 37

Malo, L., Doyon, R., Lafreniere, D., et al. 2013, ApJ, 762, 88

Mamajek, E. E. 2009, in AIP Conf. Ser. 1158, Exoplanets and Disks: Their Formation and Diversity: Pro. Int. Conf., ed. T. Usuda, M. Tamura \& M. Ishii (Melville, NY: AIP), 3

Mamajek, E. E., \& Bell, C. P. M. 2014, MNRAS, 445, 2169

Mamajek, E. E., Lawson, W. A., \& Feigelson, E. D. 1999, ApJL, 516, L77

Manara, C. F., Beccari, G., da Rio, N., et al. 2013, A\&A, 557, 114

Mayne, N. J., \& Naylor, T. 2008, MNRAS, 386, 261

Murphy, S. J., Lawson, W. A., \& Bessell, M. S. 2010, MNRAS, 406, L50

Murphy, S. J., Lawson, W. A., Bessell, M. S., \& Bayliss, D. D. R. 2011, MNRAS, 411, L51

Murphy, S. J., Lawson, W. A., \& Bessell, M. S. 2013, MNRAS, 435, 1325

Naylor, T. 2009, MNRAS, 399, 432

Pecaut, M. J., \& Mamajek, E. E. 2013, ApJS, 208, 9

Pecaut, M. J., Mamajek, E. E., \& Bubar, E. J. 2012, ApJ, 746, 154

Preibisch, T. 2012, RAA, 12, 1

Preibisch, T., Brown, A. G. A., Bridges, T., Guenther, E., \& Zinnecker, H. 2002, AJ, 124, 404

Rebull, L. M., Padgett, D. L., McCabe, C.-E., et al. 2010, ApJS, 186, 259
Reggiani, M., Robberto, M., da Rio, N., et al. 2011, A\&A, 534, 83

Riedel, A. R., Finch, C. T., Henry, T. J., et al. 2014, ApJ, 147, 85

Rieke, G. H., \& Lebofsky, M. J. 1985, ApJ, 288, 618

Rigliaco, E., Natta, A., Randich, S., Testi, L., \& Biazzo, K. 2011, A\&A, 525,47

Siess, L., Dufour, E., \& Forestini, M. 2000, A\&A, 358, 593

Skrutskie, M. F., Cutri, R. M., Stiening, R., et al. 2006, AJ, 131, 1163

Slesnick, C. L., Carpenter, J. M., \& Hillenbrand, L. A. 2006, AJ, 131, 3016

Slesnick, C. L., Hillenbrand, L. A., \& Carpenter, J. M. 2008, ApJ, 688, 377

Soderblom, D. R., Hillenbrand, L. A., Jeffries, R. D., Mamajek, E. E., \& Naylor, T. 2014, in Protostars and Planets VI, ed. H. Beuther et al. (Tucson, AZ: Univ. Arizona Press), 219

Somers, G., \& Pinsonneault, M. H. 2014, ApJ, 790, 72

Stassun, K. G., Feiden, G. A., \& Torres, G. 2014, NewAR, 60, 1

Tognelli, E., Prada Moroni, P. G., \& Degl'Innocenti, S. 2011, A\&A, 533, 109

Tottle, J., \& Mohanty, S. 2015, ApJ, 805, 57

van Leeuwen, F. 2007, A\&A, 474, 653

Weinberger, A. J., Anglada-Escudé, G., \& Boss, A. P. 2013, ApJ, 762, 118

Weingartner, J. C., \& Draine, B. T. 2001, ApJ, 548, 296

Williams, M. J., Bureau, M., \& Cappellari, M. 2010, MNRAS, 409, 1330

Zacharias, N., Finch, C. T., Girard, T. M., et al. 2013, AJ, 145, 44 